\title{
Monte Carlo Techniques for the Comprehensive Modeling of Isotopic Inventories in Future Nuclear Systems and Fuel Cycles
}

\author{
Final Report for the Period July 2002 - June 2005
}

Date IssuedPublished July 2005

\author{
Paul P.H. Wilson
}

Department of Engineering Physics

University of Wisconsin-Madison

PREPARED FOR THE UNITED STATES

DEPARTMENT OF ENERGY

OFFICE OF NUCLEAR SCIENCE, ENGINEERING AND TECHNOLOGY

Work Performed Under Contract No. DE-FG07-02ID14342 


\title{
Monte Carlo Techniques for the Comprehensive Modeling of Isotopic Inventories in Future Nuclear Systems and Fuel Cycles
}

\author{
Paul P.H. Wilson
}

\section{Executive Summary}

The development of Monte Carlo techniques for isotopic inventory analysis has been explored in order to facilitate the modeling of systems with flowing streams of material through varying neutron irradiation environments. This represents a novel application of Monte Carlo methods to a field that has traditionally relied on deterministic solutions to systems of first-order differential equations. The Monte Carlo techniques were based largely on the known modeling techniques of Monte Carlo radiation transport, but with important differences, particularly in the area of variance reduction and efficiency measurement. The software that was developed to implement and test these methods now provides a basis for validating approximate modeling techniques that are available to deterministic methodologies.

The Monte Carlo methods have been shown to be effective in reproducing the solutions of simple problems that are possible using both stochastic and deterministic methods. The Monte Carlo methods are also effective for tracking flows of materials through complex systems including the ability to model removal of individual elements or isotopes in the system. Computational performance is best for flows that have characteristic times that are large fractions of the system lifetime. As the characteristic times become short, leading to thousands or millions of passes through the system, the computational performance drops significantly. Further research is underway to determine modeling techniques to improve performance within this range of problems. 


\section{Monte Carlo Techniques for the Comprehensive Modeling of Isotopic Inventories in Future Nuclear Systems and Fuel Cycles}

This report describes the technical development of Monte Carlo techniques for isotopic inventory analysis. The primary motivation for this solution methodology is the ability to model systems of flowing material being exposed to varying and stochastically varying radiation environments. The methodology was developed in three stages: analog methods which model each atom with true reaction probabilities (Section 2), non-analog methods which bias the probability distributions while adjusting atom weights to preserve a fair game (Section 3), and efficiency measures to provide local and global measures of the effectiveness of the non-analog methods (Section 4). Following this development, the MCise (Monte Carlo isotope simulation engine) software was used to explore the efficiency of different modeling techniques (Section 5).

\section{Importance of Monte Carlo Isotopic Inventory}

Many of the current nuclear power systems rely on the fuel cycles in which material is exposed to a small number of different irradiation environments over long time scales with little to none on-line chemical processing. However, the fuel cycle's characteristic for the future system is gradually changing as some of the ongoing developments for the fuel cycles tend to be more "dynamic" as the material is anticipated to mix and circulate throughout the system and, therefore, exposed to a wide range of neutron spectrums over much shorter time scales. In addition, it is possible to integrate an on-line chemical process as a component of the system. Aside from improving overall 
performance of the system, such changes are typically introduced with the purpose of minimizing the volume, activity and/or mean half-life of the radioactive waste disposal. As a result, the amount of a high-level waste repository can eventually be decreased. Furthermore, such changes often raise concerns about an increase in the proliferation risk of the system even though no consideration has been given to the intrinsic proliferation barriers that may also be part of the new systems. Both aspects are sensitive issues that need to be addressed before the future nuclear power systems become more viable options of global energy resource.

Accurate determination of isotopic inventory, particularly the actinide inventory, of the nuclear power systems is one of the most important inputs used to quantitatively assess their waste disposal and to evaluate their resistance to proliferation. Such calculation requires tools and methodologies that permit a transient analysis of the isotopic inventories throughout the lifetime of the system or fuel cycle. Traditional methodologies for inventory analysis focus on the conversion of a macroscopic mixture of isotope via transmutation, fission, and decay reactions. The first order ordinary differential equations (ODE) that describe the reactions for each isotope are collected into a system of equations that can be written as

$$
\dot{\vec{N}}(t)=\mathbf{A} \vec{N}(t)
$$

where $\mathbf{A}$ is a transfer matrix that represents decay, production and destruction rates of all isotopes. The general solution to equation (1) is given in terms of matrix exponential [1]:

$$
\vec{N}(t)=e^{\mathbf{A} t} \vec{N}(0)
$$

Equation (1) can be solved using a variety of methods. Several activation codes, such 
as FISPIN [2], FISPACT [3] and RACC [4], use simple time-step methods by applying a difference operator to approximate the time derivative on the left hand side of equation (1) and converting a system of first order differential equations into a system of algebraic equations. An alternative method treats the system of equations as a matrix and employs one of existing computational techniques to solve the exponential as in Equation (2). ORIGEN [5] employs Taylor's series expansion to calculate the term $e^{\mathbf{A} t}$. In addition to above methods which attempt to solve the problem as one large system of ODE's, a linear chain method which is implemented by DKR [6] and CINDER [7] breaks down the reaction tree into a number of chains such that each isotopic node has only one product. Each linear chain has its associated transfer matrix A which is bidiagonal and its analytical solution is popularly known as the Bateman equations $[1,8]$.

All of the mentioned techniques have their own limitations and advantages, depending on physical problems on which they are modeling. A highly stiff nature of a transfer matrix $\mathbf{A}$ is a major obstacle that affects accuracy and convergence of the numerical method. Furthermore, their effectiveness and accuracy become the issue of concern when they are used to simulate more complicated systems, especially ones with flowing materials. First, it is often the case in most of these methodologies to assume a finite set of isotopes produced during the simulation time. Several techniques are employed to resolve this a priori assumption, ranging from including all isotopes for which data exists, to arbitrarily applying the maximum number of isotopes in each reaction chain, to specifying the size of reaction tree based on desired accuracy and truncation of the simulation. Secondly, all of these traditional techniques are suitable for the analysis of a fixed volume of static material exposed to a steady-state, pulsed or slowly varying irradiation environment. As mentioned earlier, future nuclear systems and fuel cycles, however, is likely to have constant or 


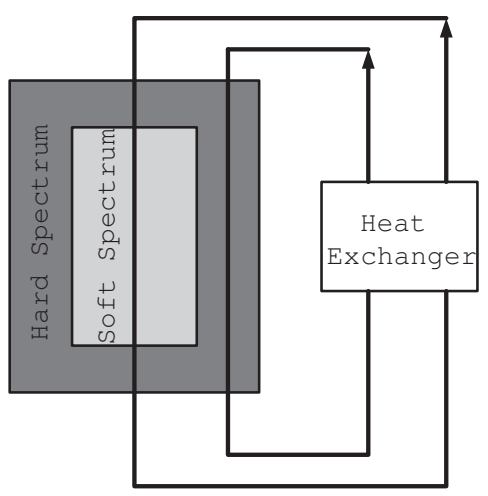

Figure 1: A simple example of mixing flow paths where two flows of coolant coming from a hard and soft spectrum region mix in the heat exchanger and re-enter the reactor.

regular addition or removal of material which causes flowing streams of fuel or other materials to experience a variety of neutron flux throughout the operating lifetime of the system. While current methodologies are capable of modeling some of these characteristics of future system to some degree of accuracy, they are clearly short of the ability to perform the computation efficiently. Finally, the implementation of flowing streams of fuel or other materials into a nuclear system suggests two supplementary features to the model: on-line chemical processes and mixing of flow paths. The on-line chemical processes would create a unique set of differential equations that must be solved concurrently with those representing transmutation and decay processes. The definition of chemical processes may be extended to include a sink in the system. Moreover, the performances of traditional techniques are susceptible to error in modeling the mixing of flow paths since flow paths can have an unpredictable effect if those flow paths previously experience different neutron fluxes. A simple reactor design as shown in Figure 1 is used as an example to illustrate a problem from using traditional techniques to approximate the problem with mixing flow paths. The reactor has coolant passing through two different regions inside and has two coolant outflows mixed in the heat exchanger. One traditional approach to approximate this 
situation is to have the same sample of coolant flowing through the hard spectrum region every time and another sample of coolant going through the soft spectrum region every time. In reality, the coolant will randomly pass through both hard and soft spectrum regions after leaving the heat exchanger. The following reaction chain that involves two consecutive transmutation reactions with energy threshold in the 1 $\mathrm{MeV}$ range is considered.

$$
A \stackrel{(n, \alpha)}{\longrightarrow} B \stackrel{(n, 2 n)}{\longrightarrow} C
$$

In this case, the approximation would overestimate the amount of $C$ in the hard region while underestimating the amount of $C$ in the soft region, with respect to its average value in the real calculation.

Aiming to overcome the drawbacks of traditional techniques, Monte Carlo techniques for modeling isotopic inventories offer the promise of modeling materials with complex flowing paths and irradiation histories. They are specifically suitable in the simulations where the arbitrary flow paths lead to non-predetermined irradiation histories.

Monte Carlo techniques which are based on tracing the history of individual atoms allow atoms to randomly follow determined flow paths, to enter/leave the system at arbitrary locations, and to be exposed to radiation or chemical processes at different portions of the flow paths. Some early potential applications of these methods include liquid breeder in fusion blankets, molten salt fueled reactor, and possibly advanced fuel cycles based on symbiotic combinations of reactors. The liquid breeder is probably the most immediate application for the use of Monte Carlo techniques. A Pb-Li coolant is used in many designs to ensure adequate tritium production. $\mathrm{Pb}$ and its impurities are subject to activation under different neutron flux environments as the coolant arbitrarily enters various regions of the reactor, e.g., first wall, shield, blanket. 
After exiting the reactor, the coolant is diverted to the heat exchanger, a chemical treatment system which is installed to extract the tritium, and back to the reactor.

Monte Carlo methods appear to have distinct advantages over traditional methodologies when a "dynamic" nuclear system is of concern. The proposed work investigates and develops a Monte Carlo approach for performing isotopic inventory analysis of dynamic material exposed to a variety of nuclear and chemical environments in a complex fuel cycle. The development begins with the implementation of a single-point steady-state calculation. The basic extensions to allow flowing and mixing of materials are followed. In addition, some enabling concepts and fundamental capabilities are described. A variety of well-known problems, some with analytic solutions and others with solutions from validated deterministic methods, are used to demonstrate the validity of the method. Finally, the methodology can be applied to the analysis of existing systems or proposed designs, including potential Generation IV reactors. Even though the realization of such nuclear systems and fuel cycles may not be practical in a number of years, early investigations of their radioactive waste disposal and proliferation resistance would certainly promote efforts in developing technology in this area.

\section{Analog Monte Carlo Isotopic Inventory}

This section introduces the methodology with specifics for implementing single point steady-state activation calculations, the first step in this development. The basic extensions to allow flowing systems are described and demonstrated. Finally, some additional enabling concepts and fundamental capabilities are shown. A variety of well-crafted problems, some with analytic solutions and others with solutions from deterministic methods, are used to demonstrate the validity of the method. Finally, 
future developments for the creation of a comprehensive tool are outlined, with a particular attention to possible variance reduction techniques.

\subsection{Methodology}

The development of this methodology follows a logical progression of complexity. First, it is necessary to develop and implement the solution for a mixture of isotopes exposed to a single steady-state flux. This is extended by adding the possibility of a simple flow path- one in which all material flows through the same sequence of control volumes- and then by allowing for complex flow paths with splitting/combining of flows. Finally, loop flow allows an atom to return to a previous control volume. Many combinations of these different flow/complexity regimes are possible. Throughout this development, a number of enabling concepts must be implemented, most importantly sources of atoms and tallies of results. This section discusses the development of both the primary methodology and its enabling concepts.

\subsubsection{Problem Formulation}

The Monte Carlo simulation of isotopic inventory is based upon following the histories of individual atoms as they pass between control volumes. An atom always has a specific isotopic identity characterized by its atomic number, mass number and isomeric state, but this identity is subject to change due to transmutation reactions and radioactive decay processes. Each control volume is characterized by a (neutron) flux ${ }^{1}$ and a residence time, $t_{\text {res }}$. The flux for each control volume, typically expressed as a multi-group spectrum, is assumed to be constant throughout the control volume. The residence time represents the average amount of time that any atom spends

\footnotetext{
${ }^{1}$ In principle, if nuclear data is available, this methodology could treat transmutation by fluxes of any type of particle and even fluxes of more than one type of particle.
} 
in the control volume. By placing an atom in a control volume, a number of new important quantities can be determined. The total effective reaction rate coefficient for an isotope $i$ in control volume $v, \lambda_{i, e f f}^{v}$, can be determined by collapsing the total transmutation cross-section for that isotope, $\sigma_{i, t o t}(E)$, with the neutron flux for that control volume, $\phi^{v}(E)$, and adding the decay constant for that isotope, $\lambda_{i, \text { decay }}$ :

$$
\lambda_{i, e f f}^{v}=\lambda_{i, \text { decay }}+\int \phi^{v}(E) \sigma_{i, t o t}(E) d E
$$

as in equation (3), $\lambda$ is a variable.

(For simplicity, the index for the isotope, $i$, and control volume, $v$, will henceforth be suppressed unless necessary for clarity.) The mean reaction time is defined as the inverse of this total effective reaction rate coefficient, $t_{m} \equiv \lambda_{\text {eff }}^{-1}$. The probability of the atom undergoing a reaction of any kind between time, $t$, and time, $t+d t$, is

$$
p(t) d t=\lambda_{e f f} e^{-\lambda_{e f f} t} d t
$$

The corresponding cumulative density function is given by integrating equation (4),

$$
\begin{aligned}
& P(t)=\int_{0}^{t} \lambda_{e f f} e^{-\lambda_{e f f} t^{\prime}} d t^{\prime} \\
& P(t)=1-e^{-\lambda_{e f f} t}
\end{aligned}
$$

At any point in time, an atom has a known amount of time before it leaves the current control volume, the remaining residence time, $0<t_{\text {rem }}\left(\leq t_{\text {res }}\right)$, and thus a remaining number of mean reaction times,

$$
n_{\text {rem }}=\lambda_{\text {eff }} t_{\text {rem }}=\frac{t_{\text {rem }}}{t_{m}}
$$




\subsubsection{Basic Elements}

\section{Steady-State Simulation}

Consider an atom that has just entered a control volume ${ }^{2}$; its remaining residence time is equal to the control volume's residence time, $t_{r e m}=t_{\text {res }}$, and its remaining number of mean reaction times is defined by equation (6). The number of mean reaction times until the next reaction, $n_{\text {ran }}$, can be randomly sampled, using the inverse transformation of equation (5) with a uniform random variable between 0 and $1, \xi$ :

$$
n_{\text {ran }}=-\ln (\xi) \text {. }
$$

If $n_{\text {ran }}<n_{\text {rem }}$, the atom reacts before leaving the control volume. The remaining residence time is updated,

$$
t_{\text {rem }} \leftarrow t_{\text {rem }}-n_{\text {ren }} t_{m}
$$

a new isotopic identity is determined by randomly sampling the list of possible reaction pathways, and a new value for is calculated based on the new isotopic identity. Finally, $n_{\text {rem }}$ is updated using equation (6), and $n_{\text {ren }}$ for the next reaction is sampled using equation (7). The history continues by repeating the comparison of $n_{\text {rm }}$ and $n_{\text {rem }}$.

If $n_{\text {ran }}>n_{\text {rem }}$, the atom leaves the control volume before reacting (and in a $0-\mathrm{D}$ simulation the history is ended).

It is perhaps clear at this point that standard steady state inventory analysis can be performed with this simple $0-\mathrm{D}$ treatment. Since the physics/mathematics of inventory analysis does not introduce coupling between spatial regions, "multidimensional" steady-state problems are solved by simply performing a 0-D solution

\footnotetext{
${ }^{2}$ Note that for a 0-D analog simulation, entering and leaving the control volume represent the beginning (birth) and end (death) of the history for that atom, respectively.
} 
a)

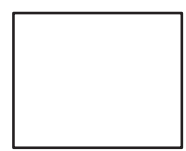

c)

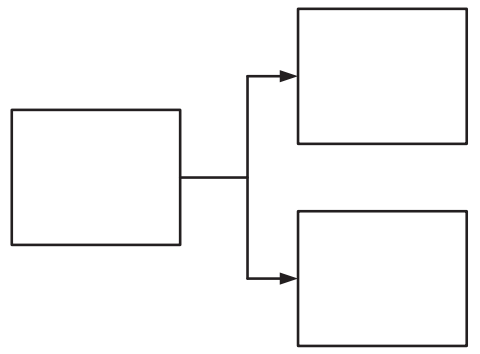

b)

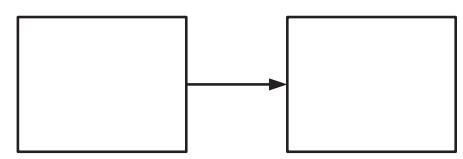

d)

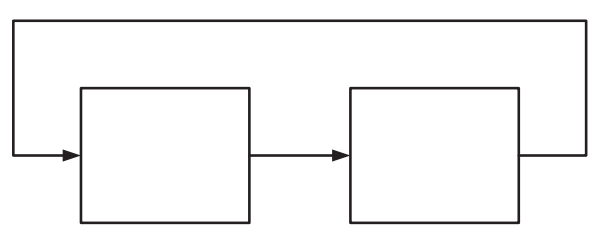

Figure 2: Four different flow and complexity regimes: a) 0-D b) simple flow c) complex flow, d) loop flow.

at each spatial point of interest in the problem.

\section{Simple Flow}

In a simple flow system, as an atom leaves one control volume, it enters another (Figure 2(b)). In this case, $n_{\text {rmn }}$ is updated

$$
n_{r m} \leftarrow n_{r m}-n_{r e m},
$$

a new value for $\lambda_{\text {eff }}$ is calculated based on the new flux in this control volume, and $t_{\text {rem }}$ is reset to the residence time for the new control volume, $t_{\text {res }}$. Finally, $n_{\text {rem }}$ is updated using equation (6). Again, the history continues by repeating the comparison of $n_{\text {ran }}$ and $n_{\text {rem }}$. Note that for simple flows, entering a new control volume requires no random sampling.

So far, time is only measured relative to the time at which a control volume is entered. It is useful to introduce a more universal definition of time, the absolute simulation time, $t_{\text {sim }}$, measured relative to some arbitrary starting time, most logically the beginning of the first control volume. This would be a property of each atom and 
be incremented each time a reaction takes place or a control volume boundary is reached. Furthermore, since the atoms now flow from one control volume to another, it is more important that the current control volume of an atom be maintained as a property of that atom. In systems with simple flows and simple sources, all atoms would originate in the first control volume at $t_{\text {sim }}=0$.

\section{Complex Flow}

In complex flow systems, atoms leaving one control volume may flow into one of any number of other control volumes - flows split and combine so that not all atoms follow the same flow path (Figure 2(c)). Implementing this is straightforward under the assumption that the relative volumetric flow rate to each control volume is known and properly characterizes the probability that a given atom will take each path. As such, random sampling of the discrete probability density function [PDF] derived from these relative flow rates fully determines which flow path a given atom takes. The absolute simulation time becomes more important here since different atoms may experience different flow paths and irradiation histories but still need to be tallied on an absolute time scale to report results.

\section{Loop Flow}

The distinguishing feature of loop flow is the ability for a given atom to return to a control volume in which it has already been resident (Figure 2(d)). Loop constructs can be combined with both simple and complex flow. If the total simulation time is being tracked properly, the implementation of loop flow does not introduce complexity to the methodology.

\subsubsection{Enabling Concepts}

\section{Atom Sources}


To this point, this discussion has quietly implied that all atoms come from the same simple source, their histories beginning in the same control volume at the same time $\left(t_{\text {sim }}=0\right)$. As such, implementation of the source would be trivial — random sampling of a discrete PDF representing the isotopic mix of the initial material. The method is rendered far more versatile; however, by accommodating different source locations, compositions and time-dependencies. In fact, the implementation is not significantly complicated by such improvements.

In its most general form, each source would be associated with a single control volume, have a single specific isotopic composition, and would have a well-defined time-dependence, $r(t)$. In a problem with many sources, the total strength of each source, $R_{s}$, would be defined by integrating the time-dependent form over the total simulation time:

$$
R_{s}=\int_{0}^{t_{s i m}^{\max }} r(t) d t .
$$

The set of total source strengths defines a discrete PDF which can be sampled to determine from which source a new atom comes. Once a particular source is chosen, its initial control volume is explicitly defined and its isotopic identity and birth time can be randomly sampled from the discrete PDF representing the isotopic mix and from the time-dependent source strength, $r(t)$,respectively. Note that the $0-\mathrm{D}$ steady state source is still supported by this generalized scheme by defining a source with a delta function time-dependence, $r(t)=R_{s} \delta\left(t_{\text {sim }}\right)$.

Further accommodations are needed to allow atoms to begin their histories at arbitrary places in a control volume, allowing a simulation to start with control volumes already containing material, some of which just entered and some of which is almost leaving. This is implemented by permitting different PDFs for the remaining residence time, $t_{\text {rem }}$, when an atom is created from a source. 


\section{Tallies}

The primary result to be estimated by tallies is the time-dependent population of atoms, possibly separated into bins based on the isotopic identity. From this result, most other quantities of interest (activity, decay heat, radiation dose) can be derived by simple scalar transformations based on nuclear data or using this result as the input to another simulation. Two types of tallies have been developed: atom current tallies that take a snapshot of the isotopic spectrum and atom population tallies that average the isotopic spectrum over a time interval.

An atom current tally simply counts the atoms as they reach user-defined points in time, but scoring in bins based upon the isotopic identity of the atom. In the simplest case, these points in time correspond to the simulation times at which atoms leave specific control volumes. In an analog simulation, every history contributes the same total score (unity) to each tally. As a result, this type of tally provides an accurate estimate of the isotopic inventory at that particular point in time but is susceptible to missing the existence of very short-lived isotopes that are both produced and consumed between two points in time. This consequence of atom current tallies is related to the analog detection limit discussed in the results of the next section.

An atom population tally is designed to counter this limitation. In such tallies, histories contribute scores to time bins, rather than points in time. Again each history contributes the same total score (unity) to each time bin in each tally. However, the total score is divided among bins that correspond to the isotopic identity of the atom during that time bin. Each isotope bin receives a score that is equal to the fraction of the time bin that the atom existed as that isotope. While this is guaranteed to detect all isotopes regardless of when they are produced or consumed, since the results are time-averaged over the width of the bins, they only estimate the results at a specific time within a discretization error of first order in time. As the number of bins becomes 


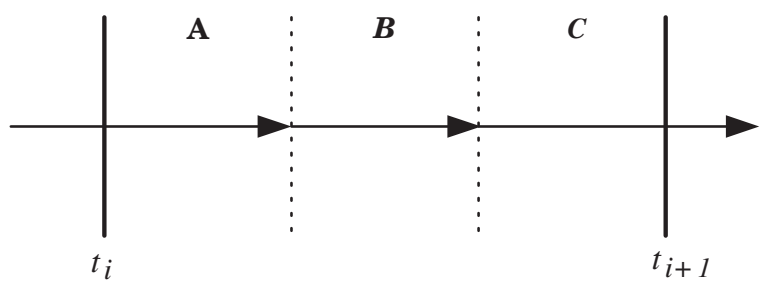

Figure 3: Representative reaction sequence between points of time to illustrate difference between current and population tallies.

\begin{tabular}{|c|c|c|c|c|c|}
\hline \hline \multirow{2}{*}{ Tally type } & \multirow{2}{*}{ Time bin } & \multicolumn{3}{|c|}{ Contribution to isotopic tally bin } & \multirow{2}{*}{ Exact time } \\
\cline { 3 - 6 } & & Isotope A & Isotope B & Isotope C & \\
\hline \multirow{2}{*}{ Current } & $i$ & 1 & 0 & 0 & $t_{i}$ \\
& $i+1$ & 0 & 0 & 1 & $t_{i+1}$ \\
\hline Population & $i$ & $1 / 3$ & $1 / 3$ & $1 / 3$ & none \\
\hline \hline
\end{tabular}

Table 1: Contributions to different tally types from sample reaction sequence shown in Figure 3.

very large within a fixed time interval, a population tally and a current tally approach the same result.

Figure 3 shows a representative reaction sequence occurring between two points in time and Table 1 indicates how each tally type would respond to this reaction sequence. None of the time bins in the current tally would include a contribution for isotope B but will be exact at the times indicated. Conversely, the population tally will not be exact at any time, but will include a contribution for isotope B.

\subsubsection{Compound Capabilities}

Taken in various combinations, the above elements and concepts can be used to derive additional capabilities. This section outlines some of these compound capabilities.

\section{Arbitrary Flow}




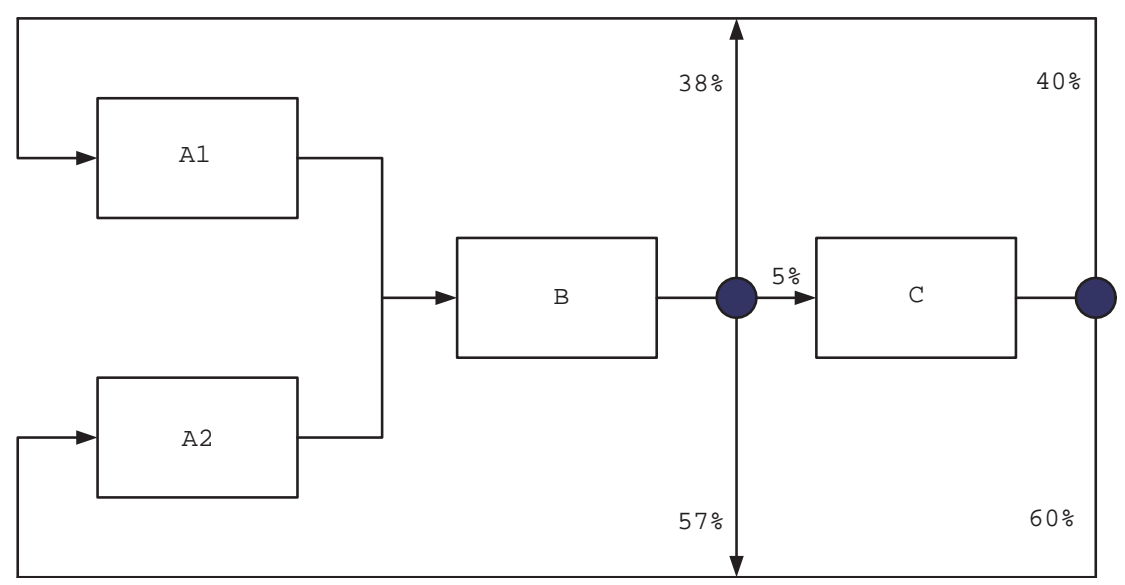

Figure 4: An arbitrary flow system (with complex and loop flows) representing a simplified two region coolant (A1 and A2) with chemical cleaning step (C) following the heat exchanger (B). In addition to the 40/60 flow split between regions A1 and A2, $5 \%$ of the flow leaving the heat exchanger is diverted to the cleanup system.

Once the basic constructs of simple, complex and loop flow have been implemented and validated, they can be combined in simulations with arbitrary complexity without adding complexity to the implementation of the methodology. A simplified schematic of a two region coolant with chemical cleanup system is shown in Figure 4. In this system, as the coolant loops through the system repeatedly, the flow through the two cooling regions is split unevenly and only $5 \%$ of the flow passes through the chemical cleanup system in each pass through the system.

\section{Atom Reservoirs: After Shutdown Decay Calculations and Atom Sinks}

In many activation calculations, the isotopic composition at various times following the shutdown of the facility is of primary importance because of its role in performing analysis of decay heat removal of waste disposal alternatives. The basic constructs of this methodology make this possible by having all material flow into a control volume with no neutron flux and a residence time that is longer than the longest cooling time of interest, an atom reservoir. A tally in this control volume at 


\begin{tabular}{|c|c|c|}
\hline \hline Element & Transport & Inventory Analysis \\
\hline Source quanta & Neutral particles & Individual atoms \\
\hline Characteristic dimension & Length of geometric cell & Residence time in control volume \\
\hline Basic sampling quanta & $\begin{array}{c}\text { Mean free paths between } \\
\text { reactions (macroscopic } \\
\text { cross-section) }\end{array}$ & $\begin{array}{c}\text { Mean times between reactions } \\
\text { (effective transmutation \& } \\
\text { decay rate) }\end{array}$ \\
\hline $\begin{array}{c}\text { Primary particle } \\
\text { characteristic }\end{array}$ & Energy & Isotopic identity \\
\hline Fundamental tallies & $\begin{array}{c}\text { Surface \& volume flux } \\
\text { (energy dependent) }\end{array}$ & $\begin{array}{c}\text { Atom current \& population } \\
\text { (Isotope dependent) }\end{array}$ \\
\hline \hline
\end{tabular}

Table 2: Analogies between elements of Monte Carlo neutral particle transport and Monte Carlo inventory analysis.

all the cooling times of interest will represent the shutdown decay inventory of the system.

Some systems might include the removal of material either at regular intervals or continuously throughout the operation period. Moreover, it may be important to simulate the instantaneous or cumulative isotopic composition of such atom sinks over the operation period. In fact, most systems with complex atom sources will require atom sinks to ensure that atom quantities of the system are conserved appropriately. This is implemented as an atom reservoir, placed anywhere in the system, representing the effluent of a chemical processing step, possible diversionary pathway or some other extraction process.

\subsubsection{Relationship to Monte Carlo neutral particle transport}

Many of the elements of Monte Carlo inventory analysis have natural analogs in the field of Monte Carlo neutral particle transport [9-12].

Table 2 highlights the most important of these. 


\subsection{Testing}

This section describes a variety of cases that have been designed to test and demonstrate the operation of the methodology described in the previous section. Following a fundamental test with a simple decay problem, a numerical benchmark for 0-D steady state problem was used as the foundation for testing each of the basic elements and enabling concepts. The results of the ALARA activation code were used as the reference solution for this benchmarking exercise. ALARA adaptively employs a variety of exact and approximate methods to solve the matrix exponential that arises as the solution of the system of first order ordinary differential equations. Finally, some sample calculations were then performed to demonstrate the compound capabilities using the $0 \mathrm{D}$ steady state problem as a reference.

\subsubsection{Single Control Volume: 0-D Analytic and Numerical}

In order to demonstrate that the underlying methodology is valid, the simplest possible analytic test case was used: pure decay from a single isotope. Figure 5 shows the results of this comparison for $10^{10}$ Monte Carlo particles simulated for 4 half-lives where the statistical error of the results is $0.001 \%$. This simple result serves to demonstrate the fundamental validity of this technique for modeling the first order ordinary differential equations that govern isotopic inventory problems and a verification of its correct implementation.

With the basic Monte Carlo sampling technique validated, the next test case was a full transmutation and decay problem: a single isotope, ${ }^{56} \mathrm{Fe}$, irradiated for 10 years at with a steady-state 175-group (vitamin-j) neutron flux where the first seven groups fluxes are zero and the remaining group fluxes are $5 \times 10^{12} \mathrm{n} / \mathrm{cm}^{2} \cdot \mathrm{s}$. The FENDL-2/A activation library and FENDL-2/D decay library were used in the calculation. The 


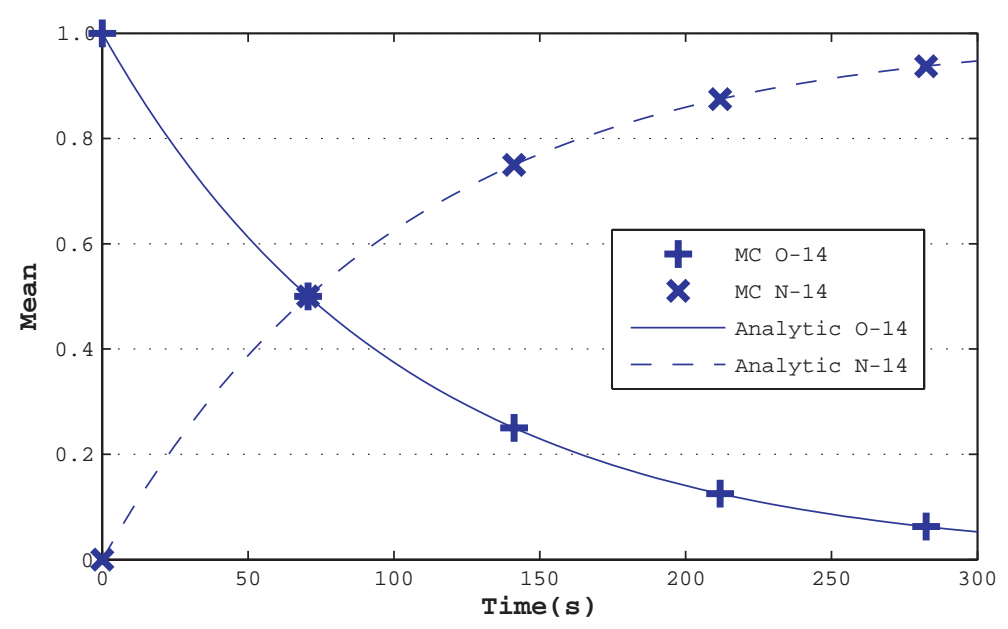

Figure 5: Comparison of Monte Carlo results to analytic benchmark results showing good agreement. Since the half life of ${ }^{14} \mathrm{O}$ was 70.60 seconds long, an initial unit amount of ${ }^{14} \mathrm{O}$ was reduced to a half and a quarter after 70.60 seconds and 141.20 seconds, respectively.

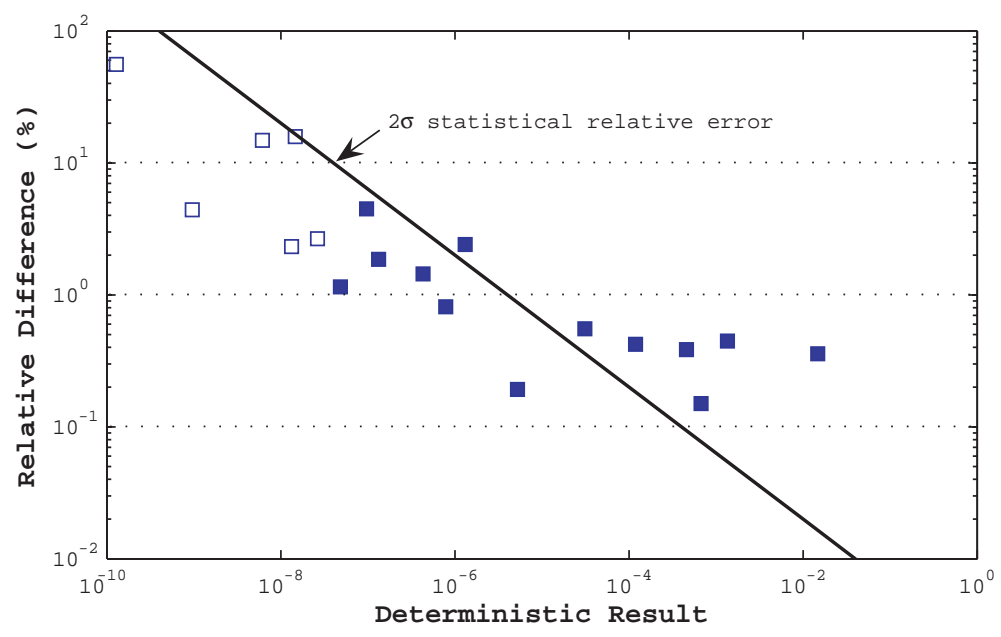

Figure 6: Variation of relative difference between Monte Carlo (MC) and deterministic results as a function of the deterministic result. The expected statistical error $(2 \sigma)$ as a function of the result is also shown for a MC problem with $10^{10}$ initial atoms. Results with a $1 \sigma$ statistical error greater than $5 \%$ are shown with open symbols $(\diamond)$. 
Monte Carlo results using $10^{10}$ atoms were compared to ALARA [1] using a truncation tolerance of $10^{-9}$.

The ALARA results included 39 isotopes whereas the Monte Carlo results only included 20. Figure 6 shows the relative difference between the Monte Carlo result and the deterministic result for 19 of these isotopes $\left({ }^{56} \mathrm{Fe}\right.$ is not included) as a function of the deterministic result itself. The line indicates the statistical error $(2 \sigma)$ associated with a result of that magnitude in an analog Monte Carlo calculation. For each isotope, the relative difference is a consequence both of the statistical variations in the Monte Carlo results and of the approximations in the deterministic calculation. The deterministic solution will include truncation errors due to the physical modeling techniques as well as an accumulation of numerical errors introduced by the mathematical methods. This representation permits some qualitative assessment of the relative contribution of these two sources of discrepancy. For seven of the isotopes, the relative difference is larger than the $2 \sigma$ statistical error, with relative differences ranging from $0.15 \%$ to $2.41 \%$. In this case, the relative difference is most likely dominated by modeling discrepancies. The remaining 12 isotopes have results with relative differences that are less than the statistical error. Of these, six have statistical errors that indicate they are statistically credible results $(2 \sigma<10 \%)$. For these isotopes, the difference is most likely dominated by the statistical variations in the results, suggesting that smaller relative differences could result from improved statistics, i.e. more initial atoms.

This hypothesis was tested by performing the same calculation with 32 times as many initial atoms. In particular, there are five isotopes that represent the intersection between those isotopes that have statistically credible results $(2 \sigma<10 \%)$ and those that have a relative difference greater than $1 \%$. If the relative difference for these isotopes does not decrease, it would indicate a potentially unreasonable differ- 


\begin{tabular}{|c|c|c|c|c|}
\hline \hline \multirow{2}{*}{ Isotope } & \multicolumn{2}{|c|}{$10^{10}$ MC Atoms } & \multicolumn{2}{|c|}{$32 \times 10^{10}$ MC Atoms } \\
\cline { 2 - 5 } & $\begin{array}{c}\text { Relative } \\
\text { Difference }\end{array}$ & $\begin{array}{c}\text { 2 } \sigma \text { Statistical } \\
\text { Error }\end{array}$ & $\begin{array}{c}\text { Relative } \\
\text { Difference }\end{array}$ & $\begin{array}{c}\text { 2 } \sigma \text { Statistical } \\
\text { Error }\end{array}$ \\
\hline${ }^{50} \mathrm{Ti}$ & $1.86 \%$ & $5.6 \%$ & $1.18 \%$ & $0.97 \%$ \\
\hline${ }^{56} \mathrm{Mn}$ & $4.49 \%$ & $6.3 \%$ & $0.65 \%$ & $1.1 \%$ \\
\hline${ }^{54} \mathrm{Fe}$ & $2.41 \%$ & $1.7 \%$ & $0.46 \%$ & $0.31 \%$ \\
\hline${ }^{59} \mathrm{Co}$ & $1.49 \%$ & $3.0 \%$ & $1.2 \%$ & $0.54 \%$ \\
\hline${ }^{60} \mathrm{Co}$ & $1.15 \%$ & $9.1 \%$ & $1.28 \%$ & $1.6 \%$ \\
\hline \hline
\end{tabular}

Table 3: Comparing improvements in results (relative to deterministic calculation) and statistical error with increasing number of initial atoms.

ence between the techniques. Table 3 shows how both the relative difference and the statistical error change with an increased number of initial atoms. Two isotopes, ${ }^{54} \mathrm{Fe}$ and ${ }^{56} \mathrm{Mn}$, converge to relative differences less than $1 \%$. Two more isotopes, with relative differences initially between $1 \%$ and $2 \%,{ }^{59} \mathrm{Co}$ and ${ }^{50} \mathrm{Ti}$, converge to relative differences that remain above $1 \%$ and are larger than the $2 \sigma$ statistical error. The last isotope, ${ }^{60} \mathrm{Co}$, converges to a relative difference greater than $1 \%$ but still less than its statistical error. This suggests that the modeling discrepancy could be as large as $1.2 \%$ for some isotopes.

Figure 7 shows how the ratio between the Monte Carlo and the deterministic results, averaged over all 20 isotopes, varies with increasing Monte Carlo sample size. For each sample size, the average ratio for all isotopes is shown. The statistical error of this average is calculated by propagating the individual statistical errors using the standard root-mean-squared summation, and is shown with error bars representing $1 \sigma$ statistical error (68\% confidence). Such a plot demonstrates the (expected) steadily improving precision of the Monte Carlo results even if the mean value does not change monotonically.

Returning to the isotopes missing from the Monte Carlo results, it is important to note that their production rate according to ALARA is less than $10^{-10}$ in all cases. 


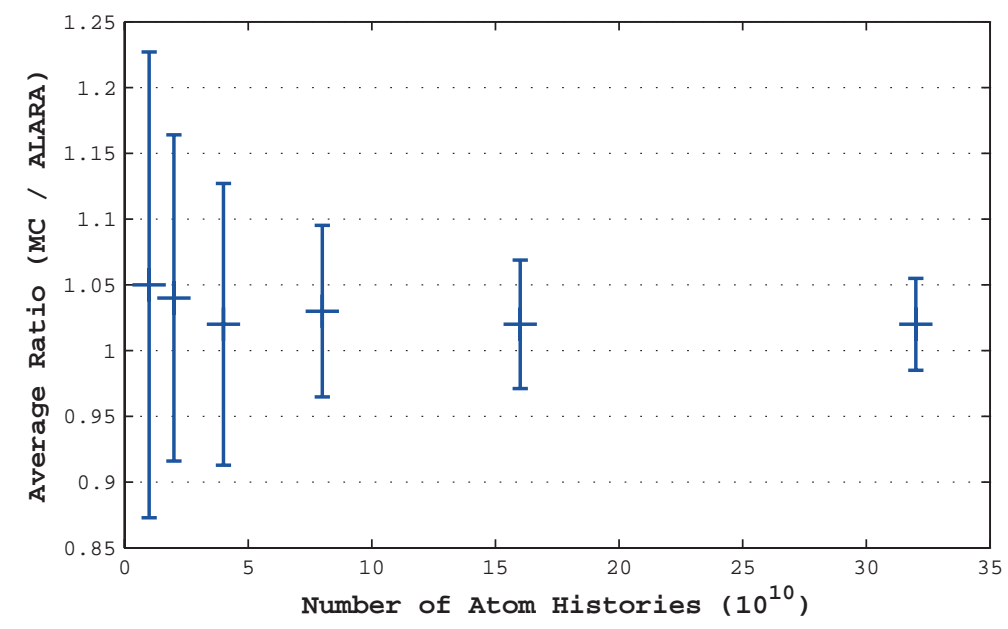

Figure 7: The average ratio (averaged over all isotopes) improves with more atom histories. The statistical error of the average is the root-mean-squared average of the individual statistical errors and is shown here as a $1 \sigma$ statistical error.

This draws attention to a fundamental limitation of atom current tallies for the analog Monte Carlo methodology - a raw detection limit that is the inverse of the number of $\mathrm{MC}$ atoms, $N$, and a statistically significant detection limit (assuming a goal of a relative statistical error, $R<5 \%$ ) of $400 / N$. The relative statistical error for the tally of a given isotope, $j$, can be derived as

$$
R_{j}=\sqrt{\frac{\sum_{i=1}^{N} x_{i j}^{2}}{\left(\sum_{i=1}^{N} x_{i j}\right)^{2}}-\frac{1}{N}},
$$

and by the definition of analog Monte Carlo, the score contribution to isotope $j$, $x_{i j}$, from a given sample atom, $i$, is 1 if that atom is the isotope in question and 0 otherwise. By defining the yield, $Y_{j}$, as the probability of producing isotope $j$ from a 
source atom, this can be reduced to

$$
\begin{aligned}
R_{j} & =\sqrt{\frac{Y_{j} \cdot N}{\left(Y_{j} \cdot N\right)^{2}}-\frac{1}{N}} \\
& =\sqrt{\frac{1}{N}\left(\frac{1}{Y_{j}}-1\right)} .
\end{aligned}
$$

Since the goal is to determine the detection limit for rare product isotopes, $Y_{j} \ll 1$, and

$$
R_{j} \approx \sqrt{\frac{1}{Y_{j} \cdot N}} \Rightarrow Y_{j} \approx \frac{1}{N \cdot R_{j}^{2}} \Rightarrow N \approx \frac{1}{Y_{j} \cdot R_{j}^{2}}
$$

Statistical error of less than $5 \%$ for results with yields of $10^{-9}$ requires $\mathrm{N}=4 \times 10^{11}$ for an analog implementation. Moreover, if an important product isotope derives only from a single isotope, $k$, that has a small relative initial concentration, $C_{k}$, the statistically significant detection limit for this resultant isotope is reduced to $C_{k} \cdot\left(N \cdot R_{j}^{2}\right)^{-1}$. Fortunately, variance reduction techniques are available to improve this situation and this methodology is well-suited to parallelization; both will later be explored.

\subsubsection{Simple, Complex and Loop Flow}

A steady-state problem with a single control volume (CV) can be duplicated by a steady-state problem with two CVs in series (Figure 8(S1)), provided they each have identical neutron fluxes and the two residence times add to the same residence time of the single CV problem (Figure 8(B)). The same is true for $10 \mathrm{CVs}$ in series each with $10 \%$ of the single CV residence time (Figure $8(\mathrm{~S} 2)$ ). This is the strategy for testing the simple flow capability.

If the second $\mathrm{CV}$ is replaced by two CVs in parallel (Figure $8(\mathrm{C} 1, \mathrm{C} 2)$ ), each with the same neutron flux and the same residence time, valid results at the end of the two 

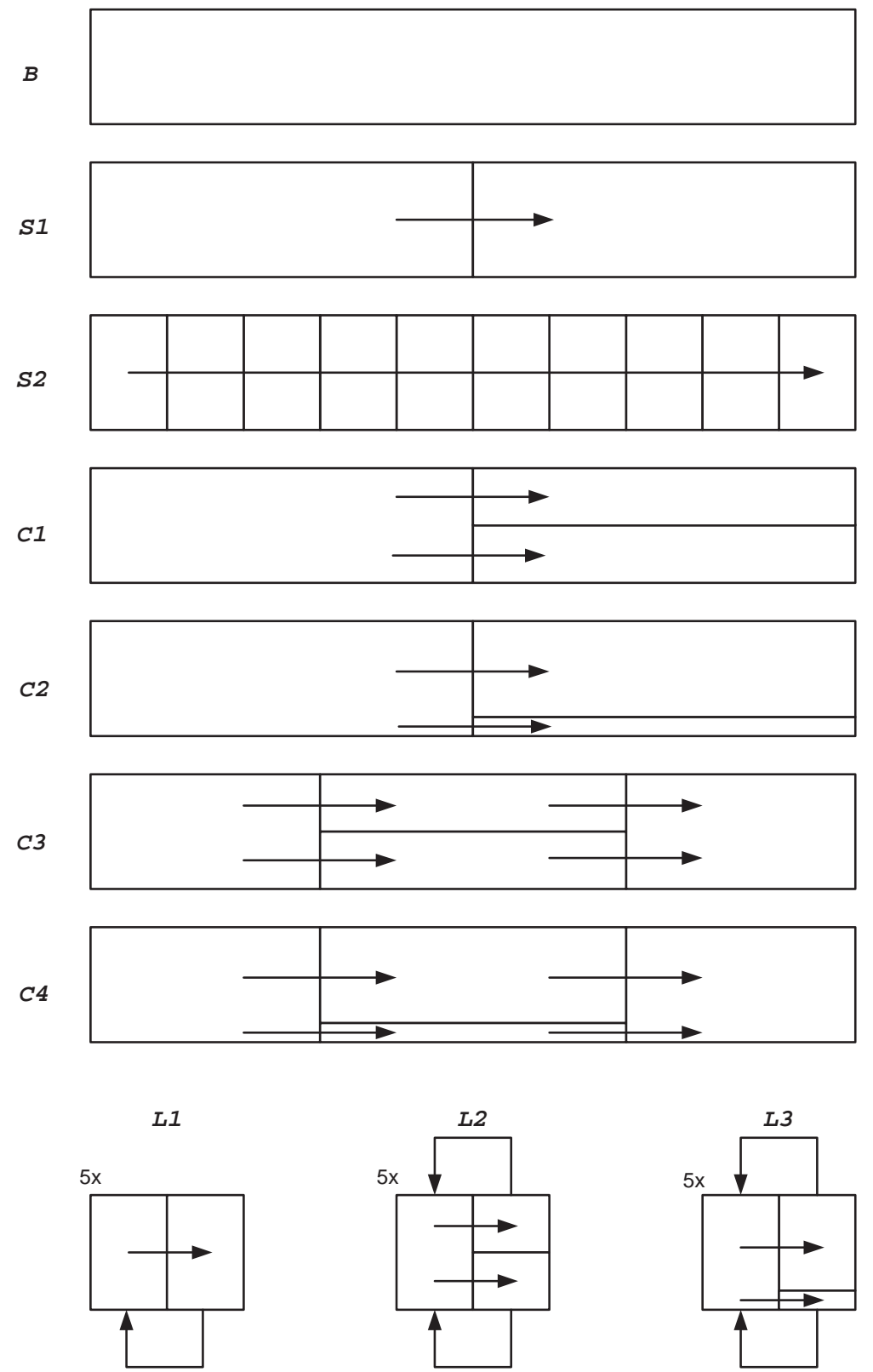

Figure 8: Physically equivalent/comparable test cases for testing flow capabilities. 
parallel CVs will have two predictable features: a) they will sum to the same as the 1 CV results, b) their ratio to each other will be equal to the flow distribution between them. Two cases are analyzed, one with a 50/50 flow split (C1) and another with a 90/10 flow split (C2). Another test of the complex flow is achieved by having the flow split and rejoin, with a total of $4 \mathrm{CVs}$ and the total residence time through either path being identical to the residence time in the single (Figure $8(\mathrm{C} 3, \mathrm{C} 4))$. The same two flow distributions, 50/50 in $\mathrm{C} 3$ and 90/10 in C4, are used to test this model. In this case, the final results should be the same as the single CV results and independent of the flow split.

Finally, both simple and complex loop flow can be tested by looping through short residence time CVs enough times to be equivalent (or comparable) to the single 0-D base case (Figure 8(L1, L2, L3)). These three cases include a simple loop (L1), a 50/50 flow split loop (L2) and a 90/10 flow split loop (L3).

Figure 9 summarizes the results for all 10 cases, including the steady-state base case, compared to the deterministic results from ALARA and normalized for the correct solution (e.g. the case C1 [50/50 complex flow] should have a result of 0.5). In all cases, the total number of Monte Carlo atoms is $10^{10}$ and the error bars represent the $1 \sigma$ statistical error of the mean. All results are within the statistical error of the correct result. The importance of variance reduction is further demonstrated here, especially in cases C2 and L3 where the number of atoms reaching some tally points is $10 \%$ of the total due to the $90 / 10$ flow splitting. In general, however, this set of results serves to demonstrate the functionality of this method for this varied set of flow conditions. 


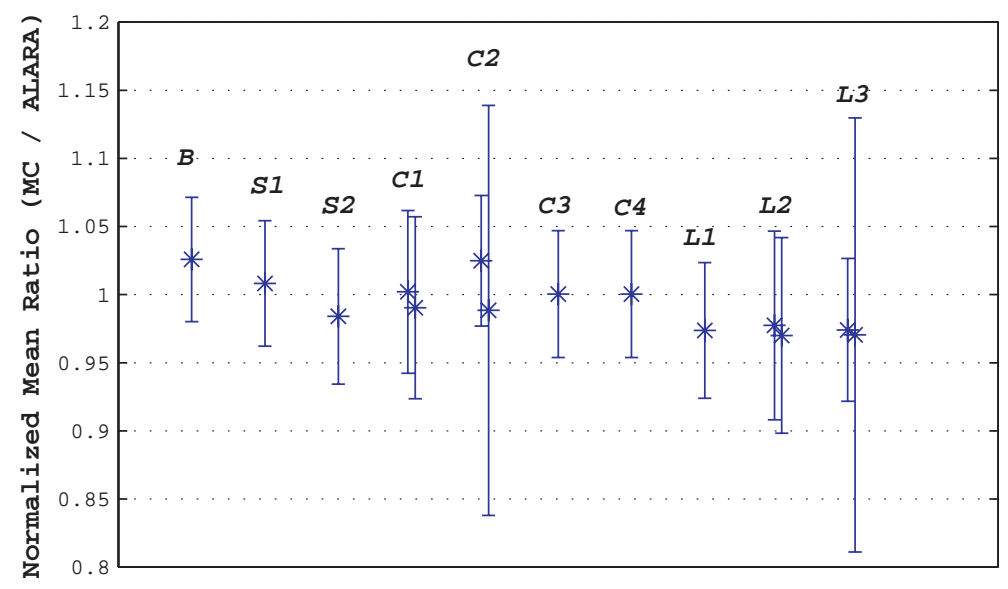

Figure 9: Summary of results for 10 cases shown in Figure 8 show that mean ratio between the Monte Carlo results and the deterministic results is within statistical error of the correct answer. The ratios have been normalized by the expected results (e.g. case $\mathrm{C} 1$ has an expected result of 0.5 ) and the error bars represent the $1 \sigma$ statistical error of that mean.

\subsubsection{Sources, Tallies, Decay and Sinks}

With the basic elements implemented and tested, some of the enabling concepts and compound capabilities can be demonstrated. The first case demonstrates sources and sinks using a variation, shown in Figure 10, of the L1 loop case. A single control volume has a residence time of two years and a steady-state flux of $5 \times 10^{12} \mathrm{n} / \mathrm{cm}^{2} \mathrm{~s}$. A fraction, $f$, of the flow leaving the control volume is diverted to a sink while the rest simply returns to the control volume. The same flow rate of source material is used to make-up the flow entering the first control volume. Note that to conserve the atom volume of the control volume, the atoms that begin in the control volume must have the same simulation time $\left(t_{s i m}=0 s\right)$ but must have their remaining residence time, $t_{\text {rem }}$, distributed uniformly throughout the two-year residence time of the control volume, allowing some to leave immediately to make room for those that are entering. 


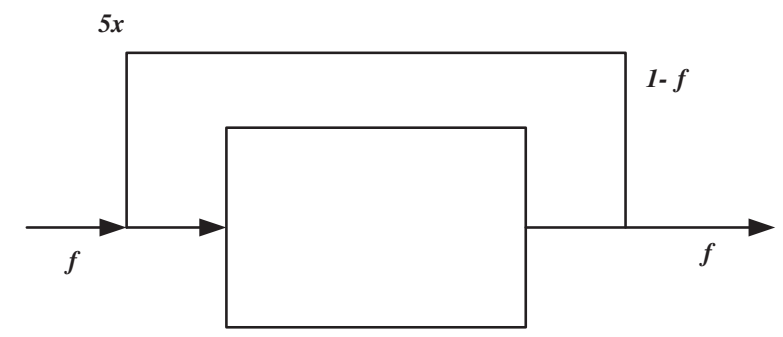

Figure 10: Test case for sources and sinks shows a fraction, $f$, of the flow being diverted to a sink and a corresponding make-up flow.

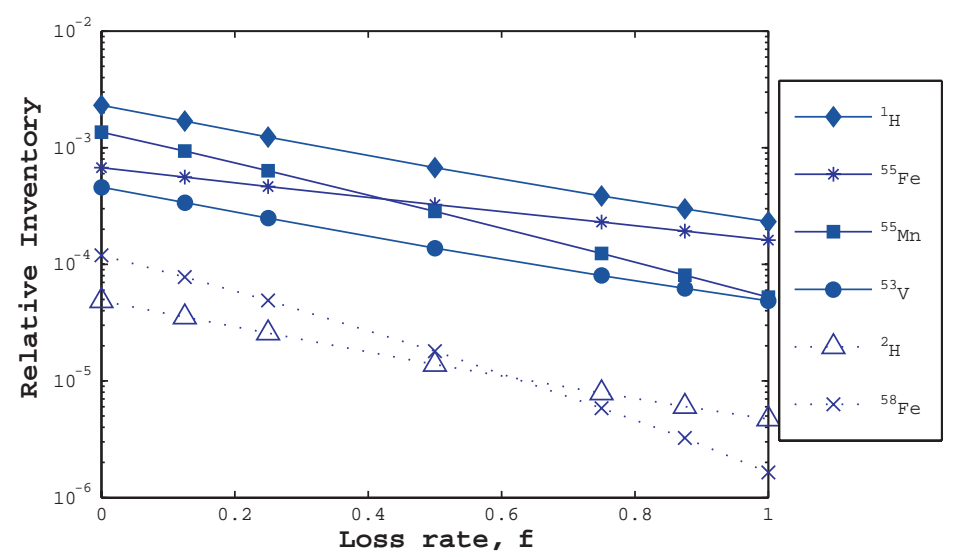

Figure 11: Relative isotopic inventories for a select sample of isotopes. Declining transmutation rates accompany a higher loss rate in source/sink problems because the average atom spends less time in the neutron flux.

Figure 11 shows the declining isotopic inventories of transmutation products as the loss rate, $f$, increases. Qualitatively, this is consistent with expectations since the average amount of time each atom spends in the control volume decreases with increasing loss rate.

In order to quantitatively benchmark these results, a mathematically equivalent problem can be constructed as the superposition of six more simple problems. The 
first simple problem tracks only the atoms that begin in the control volume, those with their remaining residence time, $t_{\text {rem }}$, uniformly distributed in the two-year time span of the control volume. Each of the atoms that are still in the control volume at the end of ten years has faced five decisions about whether or not to leave the system, and chosen, with probability $1-f$, to remain each time. More importantly, those that have remained have been exposed to a constant flux for 10 years, regardless of the initial value of $t_{\text {rem }}$, so that the contribution from these atoms is simply

$$
\left[g_{i}^{B}(10 y)\right](1-f)^{5}
$$

where $g_{i}^{B}(10 y)$ is the solution to the base single CV case for isotope, $i$. The other five simple problems track the atoms that are introduced during each of the five 2year periods, facing successively fewer decision points about whether or not to stay in the system. More specifically, during each of the 2-year periods, the atoms enter with the same remaining residence time, $t_{\text {rem }}=2 y$, but with a simulation time, $t_{\text {sim }}$, uniformly distributed between $2(j-1)$ and $2 j$ years, where $j=[1,5]$, and are tallied at $t_{\text {sim }}=10 y$. By simple variable transformation, this can instead be considered as five problems in which all the initial atoms have $t_{r e m}=2 y, t_{\text {sim }}$ uniformly distributed between 0 and 2 years, and tallied at $2 j$ years. Thus, the results of a single such problem with a tally at $2,4,6,8$, and 10 years, $g_{i}^{(2)}(t)$, will provide the necessary information to include these contributions. Each of the contributions is thus

$$
f \cdot\left[g_{i}^{(2)}(j \cdot 2 y)\right](1-f)^{(j-1)},
$$

where $\mathrm{j}=[1,5]$. The benchmark solution for the fully modeled loop extraction case 
shown in Figure 10 will therefore be the results of this six source superposition

$$
g_{i}^{\text {bench }}=\left[g_{i}^{B}(10 y)\right](1-f)^{5}+f \sum_{j=1}^{5}\left[g_{i}^{(2)}(j \cdot 2 y)\right](1-f)^{(j-1)} \text {. }
$$

Figure 12 shows the relative difference between the test case and the benchmark case for three different loss rates, $0,0.5$ and 1 . While numerical results $g_{i}^{B}(t)$ have been described above, a simple Monte Carlo problem was performed to generate results for the $g_{i}^{(2)}(t)$ problem. The $2 \sigma$ statistical error in this case if found by combining the $2 \sigma$ errors for the test case and the benchmark case in quadrature since both are Monte Carlo calculations. These results show the expected level of agreement, with most isotopes falling within the $2 \sigma$ error for all values of $f$.

The functionality of the two tally types was compared by performing the base case, $\mathrm{B}$, with both a current tally every 0.5 years and a population tally with 0.5 year bins. Over the 10 year simulation time, the current tally detected 20 isotopes while the population tally detected 26 . For the 20 common isotopes, the two tallies produce nominally the same results but differ in their precision depending on the half-life of the isotope in question. For relatively short-lived isotopes (those with half-lives less than $0.5 \mathrm{y}$ in this case) the population tally is more precise than the current tally, sometimes significantly. The population tally is nearly 25 times more precise than the current tally for ${ }^{56} \mathrm{Mn}$ with a $3.6 \mathrm{~h}$ half-life. For longer-lived or stable isotopes, the current tally is slightly more precise, typically by a multiple of 1.25 or less, and decreasing at later times. The six isotopes missed by the current tally are all present in the population tally at levels below $10^{-11}$ (below the detection limit) and all had half-lives below 11 minutes.

This half-life dependent behavior is a natural consequence of the tallying methods. The population tally detects all isotopes that occur within its time bin no matter 


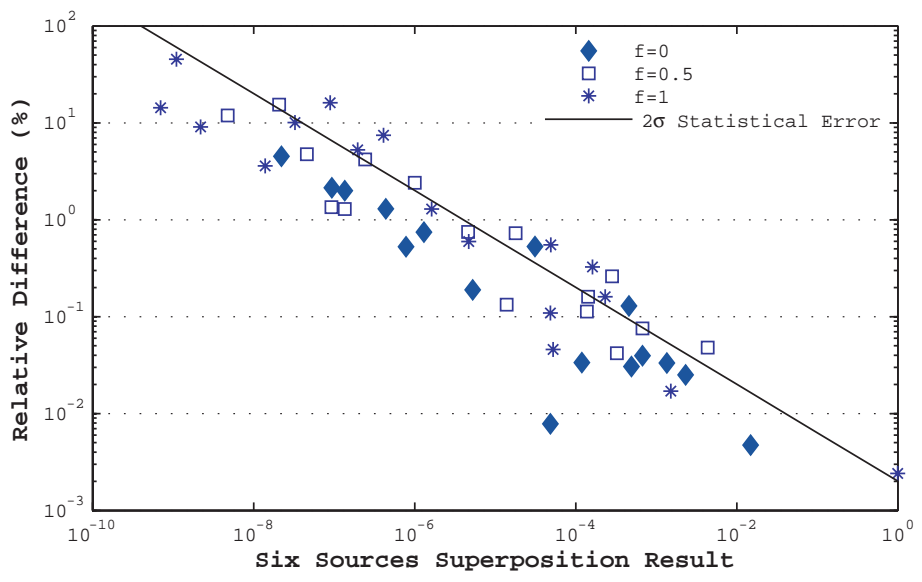

Figure 12: A comparison of relative difference as a function of the benchmark result compared to the statistical error shows agreement for values of $f=0,0.5$, and 1 .

when they occur and for how long they occur while the current tally only detects isotopes that occur when the atom crosses a certain point in time. Thus current tallies are less likely to detect the presence of a short-lived isotope. Conversely, longlived isotopes that build up slowly throughout the simulation are rare at early times and only contribute over a fraction of a time bin to population tallies while they contribute a uniform (unit) amount to current tallies. Even if the same number of atoms is detected by the two tallies, at early times the population tally will have a more statistically diverse set of contributions than the current tally.

Finally, the ability to model post-irradiation decay time, where the isotopes are not subjected to a flux was demonstrated by adding a 100-year cooling time to the base case, B. This is implemented simply as a control volume with uniformly zero flux and a 100-year residence time. Figure 13 shows the relative difference between the MC results and the deterministic (ALARA) results, as a function of the deterministic result, for three times: shutdown, 10 years post-shutdown and 100 years post-shutdown. 


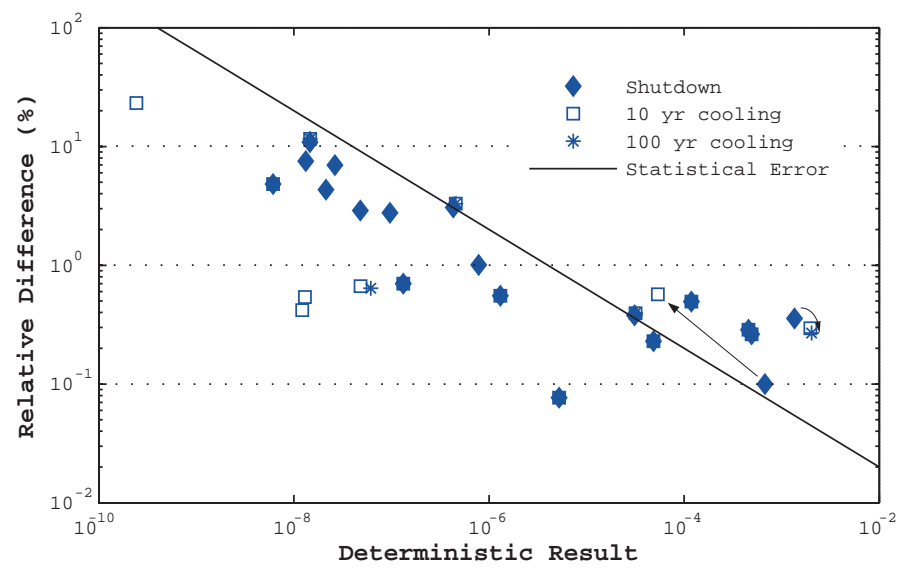

Figure 13: Relative difference as a function of deterministic result for shutdown and two difference cooling times for a 10 -year steady-state irradiation of ${ }^{56} \mathrm{Fe}$. Two arrows demonstrate the decay of ${ }^{55} \mathrm{Fe}$ resulting in the accumulation of ${ }^{55} \mathrm{Mn}$.

The results are consistent with the previous analyses. All results are either within $1 \%$ of the deterministic result or within $2 \sigma$ statistical error of the deterministic result. Additionally, however, the impact of the statistical error in closely matching results can be seen in the simple decay paths. Figure 13 includes an indication of the decay of ${ }^{55} \mathrm{Fe}$ to ${ }^{55} \mathrm{Mn}$ in this representation. One arrow shows the decay of ${ }^{55} \mathrm{Fe}\left(t_{\frac{1}{2}}=\right.$ 2.73 years) over the first 10 years (the Monte Carlo results do not include any ${ }^{55} \mathrm{Fe}$ after 100 years) while the other arrow shows the accumulation of additional ${ }^{55} \mathrm{Mn}$ as a result of this decay. In both cases, the relative difference between the MC results and the deterministic result changes as the inventories change, most clearly in the ${ }^{55}$ Fe case. Pure (deterministic) exponential decay based on the same decay constant, would result in a relative difference that is independent of time. The changing relative difference here reflects the role of the statistical uncertainty as a contribution to the total relative difference. As the decay occurs, the contribution from statistical uncertainty changes while the contribution from the modeling differences is likely unchanged. 


\begin{tabular}{|c|c|c|c|c|}
\hline \hline $\begin{array}{c}\text { Number of } \\
\text { Processors }\end{array}$ & $\begin{array}{c}\text { Wall Time } \\
\text { (seconds) }\end{array}$ & $\begin{array}{c}\text { CPU Time } \\
\text { (seconds) }\end{array}$ & Speedup & $\begin{array}{c}\text { Efficiency } \\
\text { (percent) }\end{array}$ \\
\hline 1 & 941.496 & 941.496 & & \\
\hline 2 & 475.556 & 951.070 & 1.980 & 98.989 \\
\hline 4 & 241.665 & 966.370 & 3.896 & 97.397 \\
\hline 8 & 122.296 & 977.347 & 7.699 & 96.231 \\
\hline 10 & 98.553 & 984.341 & 9.553 & 95.532 \\
\hline \hline
\end{tabular}

Table 4: Summary of computing efficiency as different numbers of processors were used on the same problem. Nearly $100 \%$ parallel efficiency can be achieved for problems of fixed total work.

\begin{tabular}{|c|c|c|c|}
\hline \hline $\begin{array}{c}\text { Number of } \\
\text { Processors }\end{array}$ & $\begin{array}{c}\text { Number of Atoms } \\
\left(10^{6} \text { particles }\right)\end{array}$ & $\begin{array}{c}\text { Wall Time } \\
\text { (seconds) }\end{array}$ & $\begin{array}{c}\text { CPU Time } \\
\text { (seconds) }\end{array}$ \\
\hline 1 & 30 & 279.808 & 279.808 \\
2 & 60 & 283.064 & 566.075 \\
4 & 120 & 282.777 & 1130.767 \\
8 & 240 & 284.041 & 2271.366 \\
10 & 300 & 282.929 & 2828.671 \\
\hline \hline
\end{tabular}

Table 5: Constant run-time can be achieved for problems with fixed work per processor.

\subsubsection{Parallel Performance}

As indicated above, the analog Monte Carlo methodology has clear limits on its detection precision that can only be overcome by sampling more atoms. Fortunately, this type of problem is straightforward to parallelize with good performance. Two different approaches can be used to demonstrate this: problems of fixed total work (i.e. work per processor decreases) and problems of fixed work per processor (i.e. total work increases). Table 4 and Table 5 show the performance for each of these approaches, respectively, on a typical Linux cluster (1.4 GHz AMD Athlon, $512 \mathrm{MB}$ RAM, 100 Mbps LAN).

In both tables, wall time refers to the time that a user would wait for the code to produce results where cpu time refers to the amount of computational resources used to produce those results. Such high parallel performance allows for improved 
statistical results by simply adding more computers to a computing cluster; however, given the scaling of statistical error with the number of particles in analog Monte Carlo simulation, only modest gains can be realized in this fashion.

\subsection{Summary}

A new methodology has been developed and implemented for the simulation of changing isotopic inventories in nuclear systems, particularly those with material flowing in arbitrary paths through the system. The current tool focuses primarily on "activation type" problems where the change in isotopics is assumed to have negligible impact on the neutron flux. The current implementation includes the ability to simulate simple, complex and loop flows, and any combination of those. These advanced abilities can then be used to implement features of real systems including sources, sinks, post-irradiation decay and extraction processes.

Pure analog calculations are limited in the relative inventories they are able to detect, either absolutely or statistically. Without implementing variance reduction techniques, large numbers of source atoms must be modeled requiring either long runtimes or large parallel clusters to achieve necessary precision. Many variance reduction techniques are being explored and expected to improve the ability of this method to provide useful results in a broader range of problems.

In addition to implementing variance reduction for improved performance, future enhancements will include new capabilities including the modeling of chemical extraction processes. 


\section{Variance Reduction Techniques}

The early benchmarks of analog results with results from a well-validated activation code in various flowing schemes were found to be successful. At the early stage, using a large number of sampled atoms, which requires long runtimes or large parallel clusters, was the only mean to improve precision. It was intuitively simple as it eliminated the need to modify an existing code or exploit the physics of the problem. However, this approach was not always favorable as the computing time was linearly proportional to number of sampled atoms and the detection limit might not be improved quickly enough. An effort to develop variance reduction techniques as alternatives to improve precision has been investigated. Variance reduction techniques are applied to improve statistical precision of the analog Monte Carlo method by modifying the underlying probability distributions that govern Monte Carlo processes so that the desired effects are produced more frequently. The importance or weight of each particle must be adjusted to obtained unbiased results.

Three variance reduction techniques, forced reaction, biased source sampling and biased reaction branching, have been explored and expected to provide useful results in a broad range of problems. Each has its own unique implementation and capability in different types of problems. Accuracy and precision improvement will be thoroughly evaluated by specifically designed test problems for each variance reduction technique. However, the efficiency enhancement will not be discussed until after the appropriate efficiency measurement tool is developed.

\subsection{Forced Reaction}

In the activation analysis, isotopes with low inventories are sometimes the most concerning. The results of these isotopes are not reliable due to their production nature. 
They often occur in the reaction tree which has an isotope with a very low total effective reaction rate in front of them. Based on a current analog calculation, the Monte Carlo simulation spends an obscene amount of time to follow a history of this almost stable isotope without advancing further into the reaction tree. Even though some Monte Carlo particles manage to transmute through this isotope, a small overall contribution yields poor statistical results for all consequent isotopes. A variance reduction technique, forced reaction (FR), betters the precision of low-production isotopes by requiring an atom to undergo a specified number of reactions in a control volume. The weight is adjusted accordingly by considering how likely each of the forced reactions would be. This technique increases the contributions not only from the isotopes produced after an almost stable isotope but also from the isotopes produced after many reactions.

\subsubsection{Methodology}

The forced reactions technique is directly analogous to the forced collision technique common in Monte Carlo radiation transport methods [9]. The forced reaction technique is based on sampling the mean time needed by an atom to undergo a reaction from the conditional probability that the reaction is to occur within the remaining residence time, $t_{\text {rem }}$, of a control volume. The underlying probability distribution can be described by an exponential distribution with a parameter $\lambda_{\text {eff }}$ where $\lambda_{\text {eff }}$ is a total effective reaction rate coefficient of a current atom entering a control volume. If $t$ is a random variable that represents the amount time the atom resides before having a reaction, the probability density function is then given by

$$
f(t)=\lambda_{e f f} e^{-\lambda_{e f f} t} \quad, t \geq 0
$$


At a pre-determined reaction time, which will later be discussed, the Monte Carlo particle with an initial weight, $W_{0}$, will be split into two particles: a reacted particle and a non-reacted particle with weights, $W_{\text {reacted }}$ and $W_{\text {non }}$, respectively. Both particles have the same identity as the initial particle. The weights of both particles depend on how likely the atom will undergo a reaction and both weights are added up to be $W_{0}$.

$$
\begin{aligned}
W_{\text {reacted }} & =W_{0} \cdot P\left(t \leq t_{\text {rem }}\right) \\
& =W_{0} \cdot \int_{0}^{t_{\text {rem }}} f(t) d t \\
& =W_{0} \cdot \int_{0}^{t_{\text {rem }}} \lambda_{\text {eff }} e^{-\lambda_{\text {eff }} t} d t \\
& =W_{0}\left(1-e^{-\lambda_{\text {eff }} t}\right) \\
W_{\text {non }} & =W_{0} \cdot P\left(t>t_{\text {rem }}\right) \\
& =W_{0} \cdot \int_{t_{\text {rem }}}^{\infty} f(t) d t \\
& =W_{0} \cdot \int_{t_{\text {rem }}}^{\infty} \lambda_{\text {eff }} e^{-\lambda_{\text {eff }} t} d t \\
& =W_{0} e^{-\lambda_{\text {eff }} t}
\end{aligned}
$$

The non-reacted particle will leave the control volume without having a reaction while the reacted particle will be forced to transmute within the control volume. Its number of mean reaction times until the next reaction, $n_{\text {ran }}$, can be randomly sampled from the conditional probability density. Given that the reaction occurs within the remaining residence time of the control volume, $t_{\text {rem }}$, the probability that the atom 
will spend $t_{\text {ran }}$ before undergoing a reaction is given by:

$$
\begin{aligned}
P\left(t \leq t_{r a n} \mid t \leq t_{\text {rem }}\right) & =\frac{P\left(\left(t \leq t_{\text {ran }}\right) \cap\left(t \leq t_{\text {rem }}\right)\right)}{P\left(t \leq t_{\text {rem }}\right)} \\
& =\frac{P\left(t \leq t_{\text {ran }}\right)}{P\left(t \leq t_{\text {rem }}\right)} \quad \because t_{\text {ran }} \leq t_{\text {rem }} \\
& =\frac{1-e^{-\lambda_{\text {eff }} t_{r a n}}}{1-e^{-\lambda_{\text {eff }} t_{\text {rem }}}}
\end{aligned}
$$

If $\xi$ is a uniform random variable in $[0,1)$, the reaction time, $t_{r x n}$, can be calculated by inverting the above conditional cumulative density function.

$$
\begin{aligned}
t_{r a n} & =-\frac{1}{\lambda_{e f f}} \ln \left[1-\xi\left(1-e^{-\lambda_{\text {eff }} t_{r e m}}\right)\right] \\
n_{r a n} & =\lambda_{e f f} \cdot t_{r a n}=-\ln \left[1-\xi\left(1-e^{-\lambda_{e f f} t_{r e m}}\right)\right]
\end{aligned}
$$

The forced reaction is controlled by the forced reaction parameter, which defines how many times a reaction is forced in a given control volume. While the forced reaction parameter is still greater than zero, another forced reaction will occur and the forced reaction parameter will be decremented by one.

\subsubsection{Test Problems}

The precision(statistical error) and accuracy(difference from reference) of FR were tested by calculating the inventory at shutdown following a 10-year steady-state irradiation of ${ }^{56} \mathrm{Fe}$ with a uniform multi-group neutron flux of $5 \times 10^{12} \mathrm{n} / \mathrm{cm}^{2} \mathrm{~s}$. The results from five non-analog test cases were compared to the analytical results by ALARA using a truncation tolerance of $10^{-9}$. The test cases with $10^{10}$ atoms had different forced reaction parameters: $1,2,3,5$ and 8 . In all cases the FENDL-2/A activation library and FENDL-2/D decay library were used. 


\subsubsection{Simulation Results}

The analytical result yields 39 isotopes but only 26 isotopes are produced at levels above the absolute analog $\mathrm{MC}$ detection limit $\left(10^{-10}\right)$ and only 21 are above the $10 \%$ statistical error limit $\left(10^{-8}\right)$ whereas the Monte Carlo analog case produces 26 isotopes. As the forced reaction parameter increases from 1 to 8 in non-analog cases, very rare/deep reaction pathways are followed, producing isotopes at levels far below the relative production tolerance used to truncate the ALARA calculation, and increasing the number of isotopes from 34 to 94, respectively. For non-analog cases, the number of isotopes that have a relative statistical error less than $10 \%$ increases from 25 to 46 . Figure 14 shows the average relative differences for 26 isotopes common to all cases, with error bars representing the $2 \sigma$ statistical error of their respective means. It is important to note that based on this metric alone, the effectiveness of forced reaction technique reaches a saturation limit, in this case, when a forced reaction parameter is three. This suggests two possible scenarios. First, these 26 isotopes occur within three reactions of the ${ }^{56} \mathrm{Fe}$ root in the activation tree. Once the forced reaction parameter is greater than three, the additional forced reactions do not contribute significantly to their tallies. Second, there might be a number of isotopes with relatively low total effective reaction rate, occurring within three reactions from the initial isotope, ${ }^{56} \mathrm{Fe}$; therefore, the effects of additional force reactions greater than three are not relevant. Since there is no significant improvement after increasing force parameter beyond three, it is likely that a number of inert isotopes occur at the third ranks of the reaction tree. These species limit the depth of the reaction tree of the problem.

To illustrate the effectiveness of forced reaction technique in reducing the statistical errors of isotopic products, the sample reaction chain of ${ }^{56} \mathrm{Fe}-{ }^{53} \mathrm{Cr}-{ }^{50} \mathrm{Ti}-{ }^{49} \mathrm{Ti}$ was 


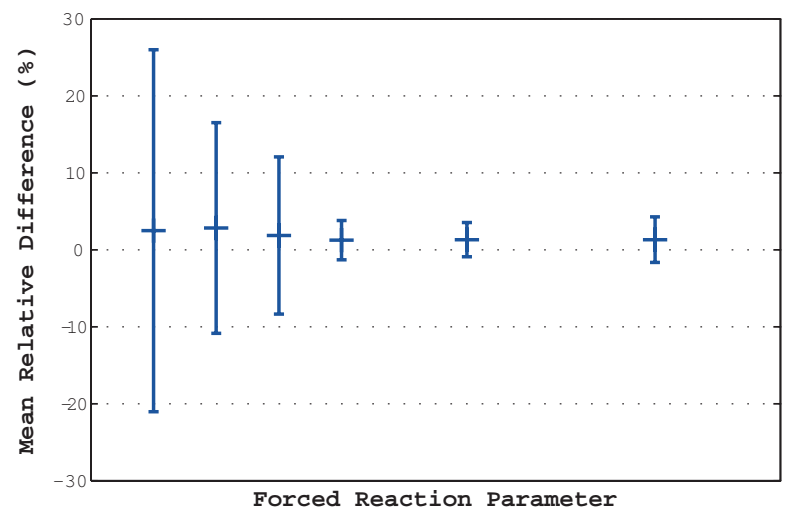

Figure 14: Average relative differences (\%) from an analog case and five non-analog cases are compared. The error bars represent $2 \sigma$ statistical errors.

investigated. Figure 15 shows the statistical errors of four isotopes as a function of the number of forced reaction. As the number of forced reaction increases, the statistical errors of the products in the chain are improved while the statistical errors of the parents are steady. When the number of forced reaction is one, ${ }^{53} \mathrm{Cr}$, which is a direct product of ${ }^{56} \mathrm{Fe}$, has its statistical error dropped about an order of magnitude while the statistical error of ${ }^{56} \mathrm{Fe}$ remains unchanged. This is due to the fact that forcing a reaction will only reduce the variance of the products in the reaction chain. For the same reason, the statistical quality of the ${ }^{53} \mathrm{Cr}$ result is not improved with additional forced reactions. Depending on its position in the reaction chain, each daughter's statistical error drops to its lowest value at different numbers of forced reactions. ${ }^{49} \mathrm{Ti}$, which is produced predominantly from the third reaction from ${ }^{56} \mathrm{Fe}$, has its variance steady after three forced reactions. This suggests that, besides increasing the number of Monte Carlo particles, other variance reduction techniques are needed to further reduce the variance. 


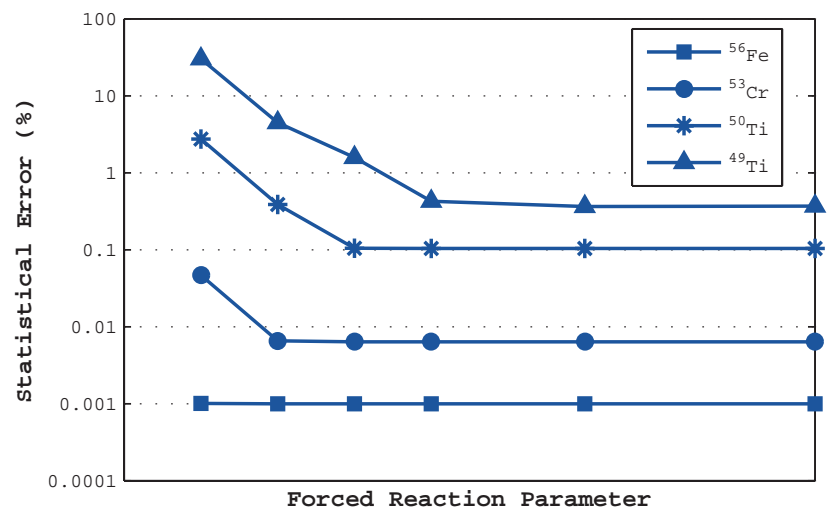

Figure 15: The statistical errors of products in one reaction chain of ${ }^{56} \mathrm{Fe}$ as a function of force parameter.

\subsection{Biased Source Sampling}

Currently, the source is implemented by randomly sampling an isotopic identity and a birth time from a probability density representing the isotopic compositions of the initial material and from a time-dependent source strength, respectively. The simplest form is the 0-D steady state source which is composed of a single isotope and the birth time is characterized by a delta function. However, it is often not the case in a real world problem, where the initial material can be composed of hundreds different isotopes. With analog source sampling, isotopes with low concentrations give way to relatively small numbers of sampled atom source, leading to statistically poor results for their respective products. The biased source sampling technique is introduced to help increasing the likelihood of sampling the rare isotopic compositions. As a result, the technique reduces the statistical errors of those products derived from rare initial isotopes. For example, the sampling mechanism can be uniform among isotopic compositions or biased in favor of a specific isotope. 


\subsubsection{Methodology}

The biased source sampling technique adjusts the importance or the weight of the starting Monte Carlo particle according to a real relative concentration and a biasing score toward the particular isotope. Let $X$ be a random variable representing an initial Monte Carlo isotope. Let $p_{i}$ and $b_{i}$ be an atomic fraction and a biasing score of the isotope $x_{i}$, respectively, $M$ be a total number of isotopic species in the initial mixture and $w_{0}$ be an initial weight of the analog starting particle. The probability of sampling the isotope $x_{i}$ with a weight, $w_{i}$, as an initial Monte Carlo isotope is given by

$$
\begin{aligned}
P\left(X=x_{i}\right)= & \frac{b_{i} p_{i}}{\sum_{j=1}^{M} b_{j} p_{j}} \\
w_{i}= & w_{0} \frac{\sum_{j=1}^{M} b_{j} p_{j}}{b_{i} p_{i}} \cdot p_{i}=w_{0} \frac{\sum_{j=1}^{M} b_{j} p_{j}}{b_{i}}
\end{aligned}
$$

It can easily be shown that the above formulations conserve the overall weight of Monte Carlo particles and realize the physical meaning of the source definition in the problem by calculating the expectations of $W$ and $W_{i}$ which represent the weight of any initial particles and the weight of a starting particle as isotope $x_{i}$, respectively. The expectation of the weight for the starting particles, $E(W)$, is found to be in agreement with analog sampling.

$$
E(W)=\sum_{j=1}^{M} w_{j} \cdot P\left(W=w_{j}\right)=\sum_{j=1}^{M} w_{j} \cdot P\left(X=x_{j}\right)
$$




$$
=w_{0} \sum_{j=1}^{M} \frac{\sum_{k=1}^{M} b_{k} p_{k}}{b_{j}} \cdot \frac{b_{j} p_{j}}{\sum_{k=1}^{M} b_{k} p_{k}}=w_{0} \sum_{j=1}^{M} p_{j}=w_{0}
$$

The expectation of $W_{i}$ corresponds to the real physical concentration of the isotope $x_{i}$ in the source multiplied by the initial weight.

$$
E\left(W_{i}\right)=w_{i} \cdot P\left(W=w_{i}\right)=w_{i} \cdot P\left(X=x_{i}\right)=w_{0} \frac{\sum_{j=1}^{M} b_{j} p_{j}}{b_{i}} \cdot \frac{b_{i} p_{i}}{\sum_{j=1}^{M} b_{j} p_{j}}=w_{0} p_{i}
$$

Uniform source sampling is a special case for biased source sampling when $b_{i}$ is equal to $1 / p_{i}$. Each isotopic composition will be sampled with equal probability, $1 / M$, and the weight is adjusted to its atomic fraction multiplied by a total number of isotopic species, $M \cdot p_{i}$.

\subsubsection{Test Problems}

The ability of biased source sampling to improve results of product isotopes transmuted from rare initial isotopes was tested by studying the inventory at shutdown following a 10-year steady state irradiation of natural iron $\left(91.75 \%{ }^{56} \mathrm{Fe}, 5.85 \%{ }^{54} \mathrm{Fe}\right.$, $2.12 \%{ }^{57} \mathrm{Fe}$ and $0.28 \%{ }^{58} \mathrm{Fe}$ ) with a uniform multi-group neutron flux of $5 \times 10^{12}$ $\mathrm{n} / \mathrm{cm}^{2} \mathrm{~s}$. Three test problems with different sampling schemes were conducted. The first problem was a pure analog test problem. The second one was a test problem with a uniform sampling technique and the third one had its source biased toward ${ }^{58} \mathrm{Fe}$, which was the least abundant isotope in the source. The test cases were simulated with $10^{10}$ Monte Carlo particles and FENDL-2/A activation library and FENDL-2/D 
decay library were used. In addition, the results were compared with deterministic results from ALARA, which solved the activation problems of each initial source isotope individually to the specified truncation tolerance of $10^{-9}$.

\subsubsection{Simulation Results}

The test problem with uniform source sampling technique applied was expected to equally distribute initial Monte Carlo particles among isotopic source compositions. Isotopes with relatively high concentrations would be chosen less frequently; therefore, the statistical errors of their respective products would be higher than results from an analog problem. At the same time, the occurrences of the rare source isotopes would be more favorable and would yield results with lower statistical errors than those from an analog problem. As a result, the overall variation in statistical errors of isotopic products would be smaller. The uniform source sampling problem produced 41 products and 27 of those had statistical errors less than $5 \%$ while the analog problem yielded 35 products with 26 products having statistical errors less than $5 \%$. Figure 16 compared the results from both problems to the deterministic results by showing the relative differences of results with statistical errors less than $5 \%$ as a function of the deterministic results. Slight improvements on both accuracy and precision were achieved as the average relative differences were found to be $0.7582 \%$ and $0.9646 \%$ and the statistical errors of the averages were $1.32 \%$ and $1.79 \%$ for uniform source sampling results and analog results, respectively.

It is important to note that uniform source sampling is simply equivalent to performing calculations on individual isotopic compositions independently and weightaveraging all of the results, according to their initial source concentrations. The technique is only useful in a problem where there is no target isotope or the overall statistical improvement is desired. 


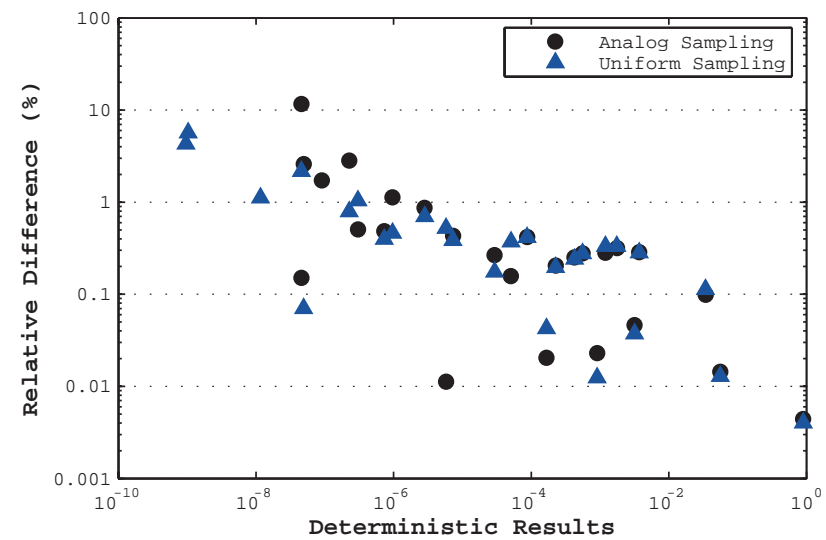

Figure 16: Relative Differences between Monte Carlo results with statistical errors less than $5 \%$ and deterministic results as a function of the deterministic result.

The last test problem was designed to evaluate the capabilities of biased source sampling. It was aimed to increase the sampling frequency of the least abundant isotope, ${ }^{58} \mathrm{Fe}$, and thus reduce statistical errors of its products. ${ }^{59} \mathrm{Fe}$ was a direct product of ${ }^{58} \mathrm{Fe}$ and had a statistical error of $1.27 \%$ from an analog problem with $10^{10}$ particles. The test problem was set up such that ${ }^{58} \mathrm{Fe}$ was likely to be sampled three times as often as the other isotopes combined. As a result, the statistical error of ${ }^{59} \mathrm{Fe}$ was reduced to $0.08 \%$ with the same number of simulated particles. However, the statistical error of about $1.20 \%$ could be achieved in the biased problem using only $5 \times 10^{7}$ particles. In this case, the computing time was reduced by about two orders of magnitude; however, the improvement of ${ }^{59} \mathrm{Fe}$ 's result came at the expense of the other isotopic results' statistical quality.

\subsection{Biased Reaction Branching}

As a simulated atom undergoes a reaction, it will be assigned a new atomic identity by sampling a discrete random variable representing a list of possible products whose probability density is characterized by the effective reaction rates for each re- 
action pathway. The product isotopes with small effective reaction rates will rarely be selected. Consequently, results with high variances for those isotopes and their respective daughters are usually obtained. Biased reaction branching is introduced to increase the occurrences of the products with low branching probabilities. The technique is based on sampling products from a modified probability density and adjusting the weights by considering how likely the products would be. Without a significant increase in the number of simulated particles, the reaction tree will become broadened and thus contain a wider range of product spectrum. The modified probability density can be uniform or biased toward specific isotopic products.

\subsubsection{Methodology}

In a control volume $v$, the branching ratio for a pair of parent isotope, $x_{i}$, and a daughter isotope, $x_{j}$, is defined as

$$
B_{i j}=\frac{\lambda_{i j, e f f}^{v}}{\lambda_{i, e f f}^{v}}
$$

where $\lambda_{i j, e f f}^{v}$ is the effective reaction rate coefficient for a reaction path, $x_{i}$ to $x_{j}$ and $\lambda_{i, e f f}^{v}$ is the total effective reaction rate coefficient for an isotope $x_{i}$. The subscript, eff, and superscript, $v$, will be suppressed for simplicity. Given the analog problem and the isotope $x_{i}$ undergoing a reaction, the branching ratio, $B_{i j}$, is indeed the probability that the isotope $x_{i}$ will transmute to the isotope $x_{j}$. It is obvious that the branching ratio is analogous to the atomic fraction during a source sampling process. In general, biased reaction branching draws a close analogy to biased source sampling with reaction products, branching ratios and the number of possible products replacing source compositions, atomic fractions and the number of initial isotopes, respectively. All of the formulations for biased source sampling are valid here with a 
weight $w_{0}$ representing a weight of the parent isotope $x_{i}$.

\subsubsection{Test Problems}

The inventories at shutdown following a 10-year steady state irradiation of ${ }^{56} \mathrm{Fe}$ with a uniform multi-group neutron flux of $5 \times 10^{12} \mathrm{n} / \mathrm{cm}^{2} \mathrm{~s}$ were calculated from three test problems with different reaction sampling schemes. The first problem was a pure analog test problem while the second problem had a uniform reaction branching technique applied and the third problem was designed to improve the results of ${ }^{53} \mathrm{Mn}$. If ${ }^{53} \mathrm{Mn}$ was present as a product, the biased reaction rates were calculated by increasing the ${ }^{53} \mathrm{Mn}$ reaction rate by a factor of ten and renormalizing the set of reaction rates. The test cases were simulated with $10^{10}$ Monte Carlo particles and FENDL-2/A activation library and FENDL-2/D decay library were used throughout. In addition, the results were compared with deterministic results from ALARA using the truncation tolerance of $10^{-9}$.

\subsubsection{Simulation Results}

The analog problem produced 26 isotopes, including ${ }^{56} \mathrm{Fe}$. Of these, 17 have statistical errors less than 5\%. When compared to deterministic results, the average relative difference of these seventeen isotopes was $0.91 \%$. As expected, the test problem with uniform reaction branching produced a much wider range of product spectrum than did the analog test problem. Sixty three isotopes were produced and 19 of these have statistical errors less than $5 \%$. The average relative difference of these isotopes was found to be $2.13 \%$. Higher deviations for low-variance results from uniform reaction branching case were due to the fact that simulated particles were uniformly distributed among possible reaction pathways, regardless of how likely they were to be sampled in an analog case. Therefore, the statistical error of those low-variance 
isotopes became higher and resulted in lower accuracy.

In particular, there were 15 identical isotopes from both cases that have statistical error less than $5 \%$. The analog problem had two isotopes that were not detected by the uniform reaction branching problem. They were ${ }^{59} \mathrm{Co}$ and ${ }^{60} \mathrm{Co}$. Because they occurred at least five reactions from the initial isotope, ${ }^{56} \mathrm{Fe}$, and the uniform reaction branching widened and implicitly shortened a reaction tree for the same number of Monte Carlo particles as the analog case. It was obvious that the analog case allowed more histories to follow the reaction pathways with high reaction rates and thus created more chances for histories to reach deep isotopes in the reaction tree than the uniform reaction branching case did. On the other hand, the latter produced four new isotopes with statistical errors less than $5 \%$. These isotopes $\left({ }^{3} \mathrm{H},{ }^{3} \mathrm{He},{ }^{51} \mathrm{~V}\right.$ and ${ }^{53} \mathrm{Mn}$ ) were produced in the analog case with extremely high statistical errors, as high as $44 \%$ for ${ }^{3} \mathrm{He}$. Obviously, those results were statistically improved in the uniform reaction branching case because the technique increased contributions from isotopes that were produced through reaction paths with low probability. The light ions, ${ }^{3} \mathrm{H}$ and ${ }^{3} \mathrm{He}$, benefitted most from the technique because they were produced sparsely throughout the reaction tree.

The next test problem was to study the effect of biasing a reaction toward ${ }^{53} \mathrm{Mn}$. The statistical error and the relative difference from a deterministic result of ${ }^{53} \mathrm{Mn}$ 's result from the analog case were $13.36 \%$ and $8.20 \%$, respectively. A larger discrepancy in the precision suggested that applying a variance reduction technique to improve precision would likely lead to a better accuracy of the result. The uniform reaction branching was then introduced to the problem. As expected, both precision and accuracy discrepancies were reduced to $3.24 \%$ and $2.68 \%$. However, the last test problem which was implemented with the biased reaction branching toward ${ }^{53} \mathrm{Mn}$ did not perform better than the uniform case as both precision and accuracy discrepancies 
became $4.92 \%$ and $7.84 \%$.

The reaction chains of ${ }^{53} \mathrm{Mn}$ from both cases were carefully examined to explain larger errors discovered in the last test problem. The test problem with uniform reaction branching produced sixty-seven reaction chains leading to the production of ${ }^{53} \mathrm{Mn}$ while the last test problem generated only five such chains which were all included in the previous case. Based on a small number of reaction chains of ${ }^{53} \mathrm{Mn}$ in the latter case, lack of accuracy and precision in ${ }^{53} \mathrm{Mn}$ 's result was a direct consequence of the technique's inability to detect the ${ }^{53} \mathrm{Mn}$ productions through reaction paths containing isotopes with relatively low total effective reaction rate coefficients. Examples of those reaction chains are given below:

$$
\begin{aligned}
& { }^{56} \mathrm{Fe} \rightarrow{ }^{56} \mathrm{Mn} \rightarrow{ }^{56} \mathrm{Fe} \rightarrow{ }^{55} \mathrm{Fe} \rightarrow{ }^{54} \mathrm{Fe} \rightarrow{ }^{53} \mathrm{Fe} \rightarrow{ }^{53} \mathrm{Mn}, \\
& { }^{56} \mathrm{Fe} \rightarrow{ }^{54} \mathrm{Mn} \rightarrow{ }^{53} \mathrm{Mn} \rightarrow{ }^{52} \mathrm{Mn} \rightarrow{ }^{53} \mathrm{Mn} \rightarrow{ }^{54} \mathrm{Mn} \rightarrow{ }^{53} \mathrm{Mn}, \\
& { }^{56} \mathrm{Fe} \rightarrow{ }^{54} \mathrm{Mn} \rightarrow{ }^{53} \mathrm{Mn} \rightarrow{ }^{54} \mathrm{Mn} \rightarrow{ }^{53} \mathrm{Mn} \rightarrow{ }^{54} \mathrm{Mn} \rightarrow{ }^{53} \mathrm{Mn}, \\
& { }^{56} \mathrm{Fe} \rightarrow{ }^{54} \mathrm{Mn} \rightarrow{ }^{55} \mathrm{Mn} \rightarrow{ }^{55} \mathrm{Cr} \rightarrow{ }^{55} \mathrm{Mn} \rightarrow{ }^{54} \mathrm{Mn} \rightarrow{ }^{53} \mathrm{Mn}, \\
& { }^{56} \mathrm{Fe} \rightarrow{ }^{55} \mathrm{Mn} \rightarrow{ }^{56} \mathrm{Mn} \rightarrow{ }^{56} \mathrm{Fe} \rightarrow{ }^{55} \mathrm{Fe} \rightarrow{ }^{54} \mathrm{Mn} \rightarrow{ }^{53} \mathrm{Mn}, \\
& { }^{56} \mathrm{Fe} \rightarrow{ }^{54} \mathrm{Mn} \rightarrow{ }^{53} \mathrm{Mn} \rightarrow{ }^{52 m} \mathrm{Mn} \rightarrow{ }^{52} \mathrm{Mn} \rightarrow{ }^{52 m} \mathrm{Mn} \rightarrow{ }^{52} \mathrm{Mn} \rightarrow{ }^{53} \mathrm{Mn} .
\end{aligned}
$$

Evidently, biasing a reaction tree toward a particular isotope does not always max- 


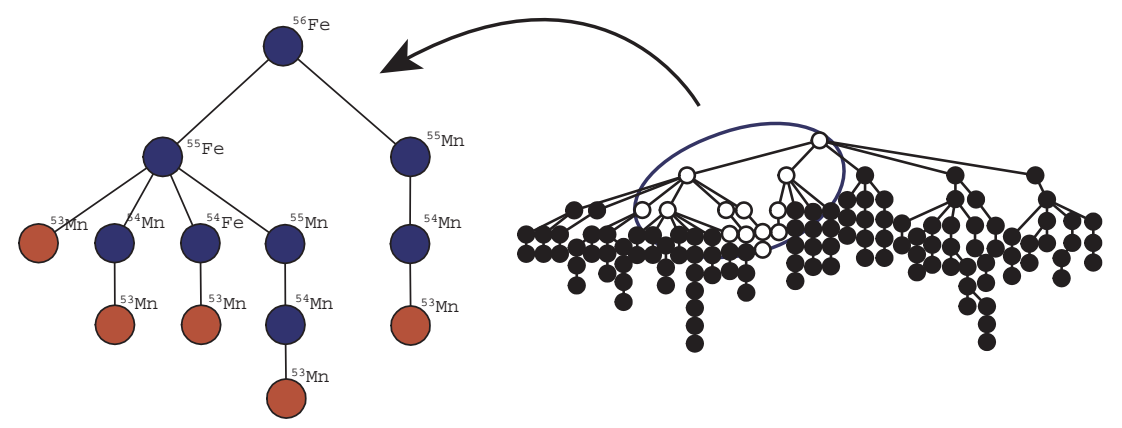

Figure 17: Most of the reaction chains leading to productions of ${ }^{53} \mathrm{Mn}$ from a uniformbranching problem, including all five chains from a biased-branching problem (o), are shown. The latter case fails to include most ${ }^{53} \mathrm{Mn}$ 's production pathways and thus results in much higher errors.

imize the accuracy and precision of its result as the technique tends to underestimate significant contributions to the isotope's inventory from other reaction pathways. The biasing scores should also be assigned to those isotopes leading to such contributions and ranged from the highest to the lowest values for the channel with maximum and minimum contributions, respectively. Defining a set of appropriate biasing scores that optimizes the accuracy and precision of the isotope in question is a research challenge and might require prior knowledge of the whole reaction tree and the extensive use of figure of merit that will later be discussed.

\subsection{Summary}

In addition to a simple increase in the number of simulated particles, variance reduction techniques are alternative methods to improve the precision of the results of isotopic inventory problems. To this point, three variance reduction techniques: forced reaction, biased source sampling and biased reaction branching, have been studied and successfully implemented. With variance reduction techniques applied, precision, accuracy and efficiency of the problem have been tremendously improved. However, using only one technique at a time might not produce the most efficient modeling 
scheme in most situations. The combination of several techniques and appropriate parameter inputs are recommended. As an example, forced reaction and biased reaction branching would be used together to both broaden and deepen a reaction tree. Nonetheless, finding suitable parameters for each technique that yields optimal performance is quite a challenging task. A figure of merit was then introduced as a tool to evaluate the efficiencies of different modeling schemes on the same physical problem, leading to the optimal variance reduction parameters.

\section{Figures of Merit}

Monte Carlo isotopic inventory analysis is directly analogous in many ways to Monte Carlo neutral particle radiation transport; instead of neutral particles traveling through 3-dimensional space and having interactions with the underlying materials that change their energy, inventory analysis simulates atoms moving through (1-D) time and having interactions with the underlying neutron flux that change their isotopic identity. After a description of the fundamental Monte Carlo methodology, including some basic variance reduction methods, and of the motivation and goals for the development of a figure of merit, this section will explore a number of possible figures of merit and discuss their applicability to different types of simulations.

\subsection{Introduction and Importance of Figure of Merit}

Even though the variance reduction techniques have tremendously reduced the statistical error and increased the spectrum of isotopes generated in the Monte Carlo simulation, they also increase the computer time spent calculating each history and thus are not guaranteed to optimize the computing resource for such calculations. That is, in some cases it may be more efficient to simply simulate more histories than 
use a variance reduction technique. A figure of merit (FOM) is introduced to monitor the efficiency of the Monte Carlo code as different variance reduction techniques are attempted on the same problem [13]. FOMs representing relative efficiencies from biased and unbiased runs of the same physical problem can be calculated and compared quantitatively, assisting a user in determining the optimal variance reduction parameters. This chapter will first consider the desired properties of a figure of merit and then introduce a number of possibilities for the figure of merit, discussing situations where each may be a useful measure.

\subsection{Principles and Constraints of Figure of Merit}

Since the underlying distribution that governs the random walk process is rarely known a priori, determining the most computationally efficient modeling scheme requires testing a variety of schemes and comparing their performance. In order to be useful, however, a quantitative metric is necessary to permit this comparison. This is the role of a figure of merit (FOM), and as such, defines the characteristics of an ideal figure of merit. In practice, a FOM will be used to determine which set of variance reduction parameters are most efficient for a given problem by comparing the FOMs that result from short problems, each with different variance reduction parameters. The set of parameters with the best FOM would be used to run a simulation with many more histories to achieve the final answer with sufficient precision. Therefore, a FOM should be a quantitative measure that is somehow proportional to the overall efficiency of the problem. This allows a user to compare two alternative modeling schemes. In addition, an ideal FOM is independent of the number of Monte Carlo histories so that a simulation with few histories can be used as an indication of the efficiency of a simulation with many histories. Finally, an ideal FOM should have a 
clear mathematical relationship to the statistical quantities used to measure the performance and one-to-one functional relationship with the computational cost. This allows a user to estimate the number of histories required to achieve the desired level of precision/performance. If the statistical performance metric, $P_{t}$, is measured over a short test time, $T_{t}$, and the performance goal is $P_{g}$, then an ideal FOM would allow a determination of the time to reach the goal, $T_{g}$, as:

$$
\begin{array}{r}
F O M=f\left(T_{t}\right) g\left(P_{t}\right)=f\left(T_{g}\right) g\left(P_{g}\right)=\text { Constant } \\
T_{g}=f^{-1}\left[\frac{F O M}{g\left(P_{g}\right)}\right]=f^{-1}\left[\frac{f\left(T_{t}\right) g\left(P_{t}\right)}{g\left(P_{g}\right)}\right]
\end{array}
$$

In summary, an ideal figure of merit has the following characteristics:

- quantitative measure of statistical performance,

- independent of number of Monte Carlo histories, and

- a one-to-one function of the computational cost.

While previous work on determining the efficiency of simulations provides a rigorous decision-theoretic framework evaluating and possibly determining the efficiency of simulations [14], this work takes an approach based more directly in the desired physical outcomes of the simulation. Future work can be carried out to reconcile the efficiency measures developed here with the mathematical development in [14].

The following sections will consider a variety of potential FOMs, both for these characteristics and for other ways that they provide information about the problems performance. 


\section{Test Problem}

The performance of each FOM was tested by calculating the proposed FOM at shutdown following a 10-year steady state irradiation of ${ }^{56} \mathrm{Fe}$ with a uniform multi-group neutron flux of $5 \times 10^{12} \mathrm{n} / \mathrm{cm}^{2} \mathrm{~s}$. In all cases the FENDL-2/A activation library and FENDL-2/D decay library were used. The results for the analog case were compared to non-analog cases with forced reaction parameters of $1,2,3,5$, and 8 . The forced reaction parameter indicates the number of times an isotope will be forced to undergo a reaction within the 10-year irradiation period. The FOM was calculated for each case as a function of time, in increments of 1000 seconds of computer time.

\subsection{Development of Figures of Merit}

Based on the above characteristics, developing a Figure of Merit requires first choosing a statistical performance metric and then examining its relationship to the computational cost to arrive at a formulation that is independent of the number of histories. A variety of statistical performance metrics are available and they form the basis for distinguishing among the four FOM formulations proposed in this section. The behavior of the specific statistical performance metric will be considered first and then its usefulness in formulating a FOM will be addressed.

\subsubsection{Statistical Error of Single Result $\left(1 / R^{2} T\right)$}

The strong analogy between this methodology and Monte Carlo neutral particle transport results in an obvious suggestion for a FOM based on the statistical error of the result. In this case, the statistical performance metric is the square of the relative statistical error, $R$, which is known to vary inversely with the number of Monte Carlo particles and hence inversely with the computational cost, T. Thus, a FOM based on 
this metric is

$$
F O M=\frac{1}{R^{2} T}
$$

Implicitly, this metric/FOM is based on the relative error of a single tally result, or single tally bin. Consequently, optimizing this FOM amounts to optimizing the results for only that tally bin and only improves the whole problem to the extent that the tally bin in question represents the rest of the problem. In the current Monte Carlo inventory implementation, the tally bins are based on time (analogous to space in Monte Carlo transport) and/or isotopic identity (analogous to energy in Monte Carlo transport). Using this FOM, therefore, requires the choice of a specific time and isotopic identity.

It is important at this stage to point out a distinct difference between the inventory analysis and the neutral particle transport methods. The energy domain of neutral particle transport is a continuous dimension where two different energies have a clear physically meaningful relationship to each other. This often allows a single energy bin (or the total over all energy bins) to be representative of the results over the entire energy domain. The isotopic identity domain is discrete and two isotopic identities may not have any clear relationship to each other. Hence a single isotopic identity bin is unlikely to be representative of many other isotopic identities, and rarely representative of the whole problem. This FOM is therefore only valuable in optimizing the modeling scheme for a particular isotope, $i$. For this study, the inventories at the end of a 10-year irradiation period were used to calculate while the FOMs, comparing results for a variety of specific represented isotopes, still remained in questions.

Since $R^{2}$ is inversely proportional to $T$ and $T$ is directly proportional to the number of histories, FOM should be constant with respect to the number of histories 
(allowing for statistical fluctuations at very small numbers of histories). Three isotopes from the test problem, ${ }^{56} \mathrm{Fe},{ }^{54} \mathrm{Cr}$ and ${ }^{59} \mathrm{Co}$, with different relative expected end-of-problem inventories of $9.82 \times 10^{-1}, 3.10 \times 10^{-5}$ and $4.29 \times 10^{-7}$, were chosen to be isotopes in question. The behaviors of the three resulting FOM values are shown in Figure 18(a-c), demonstrating the effects of increasing the forced reaction parameter. The FOM for all three isotopes are constant as expected. For ${ }^{56} \mathrm{Fe}$, this FOM suggests that the analog scheme is much more efficient than forced reactions technique. This agrees with physical nature of the problem; because ${ }^{56} \mathrm{Fe}$ is the initial isotope, forcing a reaction will only reduce the variance of the products in the reaction chain, but will make each history have a higher computational cost. On the other hand, both ${ }^{54} \mathrm{Cr}$ and ${ }^{59} \mathrm{Co}$ are produced predominantly from the third reaction from ${ }^{56} \mathrm{Fe}$ and the forced reaction technique with parameter of 3 improves the likelihood of producing these isotopes with the minimum increase in the computational cost of each history. This FOM confirms that this is the optimal choice for these isotopes.

Finally, this FOM can be used to estimate a computing time to achieve a target error for the isotope in question:

$$
T_{g}=\frac{1}{R_{g}^{2} \cdot F O M}=\frac{R_{t}^{2} T_{t}}{R_{g}^{2}}
$$

as described in Equation (26). This FOM has all of the characteristics of an ideal FOM defined above, but the performance metric itself is only useful in special circumstances. Since the important isotopes are not always known a priori and one isotope's results are rarely indicative of another's, other potential performance metrics and FOMs will be explored. 
a)

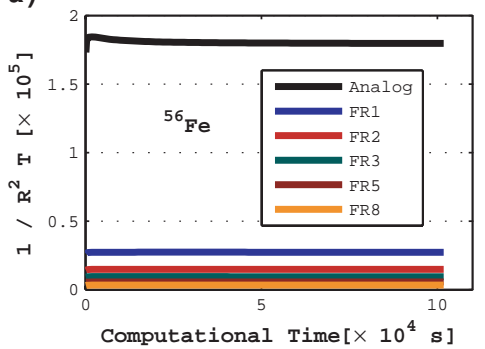

b)

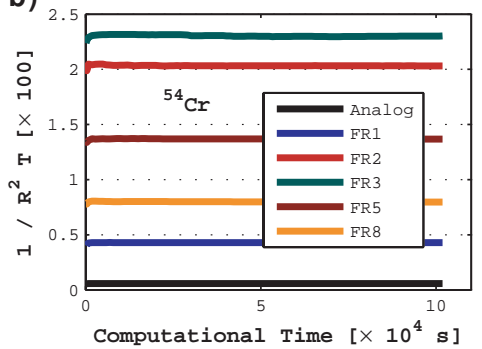

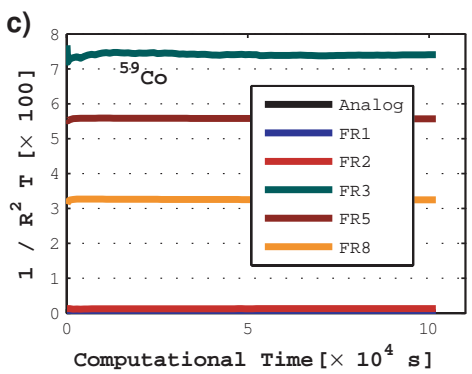

Figure 18: Traditional figure of merit based on relative error, shown for three different isotopes as a function of time and for different forced reaction(FR) parameters: a ${ }^{56} \mathrm{Fe}$ b) ${ }^{54} \mathrm{Cr} \quad$ c $)^{59} \mathrm{Co}$.

\subsubsection{Number of Qualified Isotopes $\left(N_{k}\right)$}

Another performance metric for the Monte Carlo inventory method is the number of isotopes produces by a given set of modeling parameters. If the statistical error for a particular isotope is too high; however, it should be disregarded. The number of qualified isotopes, $N_{k}$, is defined as the number of isotopes that have relative errors less than $k$ percent. This quantity is the simplest measurement of the performance for a given modeling technique.

Figure 19 shows the results for this performance metric for a $k$ of $1 \%$ and for a variety of modeling schemes represented by the forced reaction parameter. For very short run times, all cases produce approximately the same number of qualified isotopes. As the computing time progresses, Figure 19 clearly shows that forced reaction problems with higher parameter(3, 5 and 8) perform similarly, but better than problems with lower forced reaction parameters. Increasing the number of Monte Carlo 


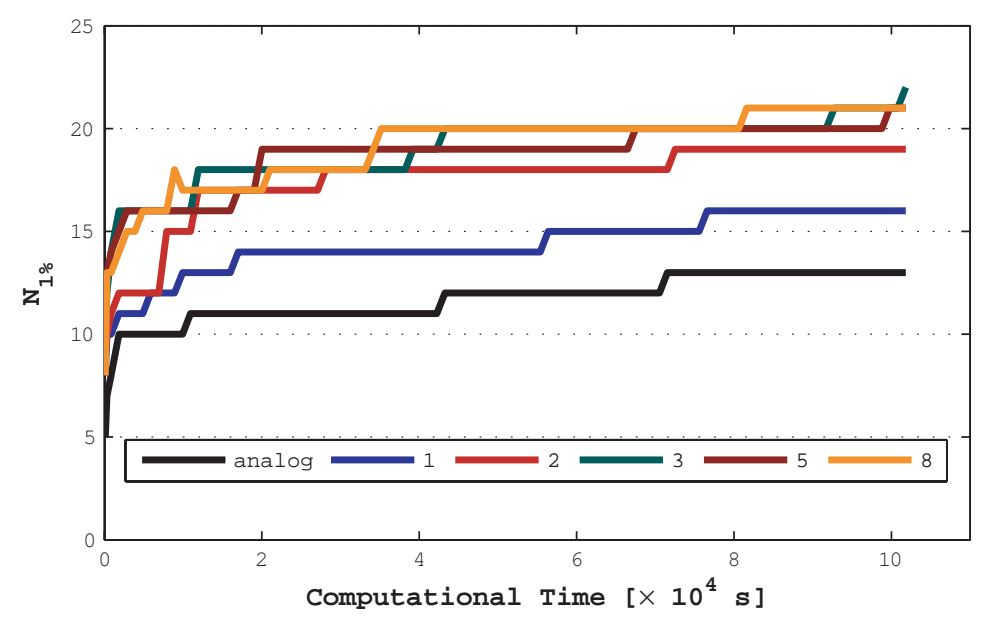

Figure 19: Number of qualified isotopes with relative error less than 1\%. The performances of the test problem with different FR parameters are compared using the number of qualified isotope metric.

particles and thus computing time would decrease relative errors of all isotopes. Consequently, more isotopes would be counted as qualified isotopes. This performance measure is expected to have this step behavior until the maximum number of isotopes is qualified, with the more efficient variance reduction schemes approaching this maximum more quickly. (In theory, the maximum number of qualified isotopes is equal to the full scope of the data library since any isotope has a finite probability of being produced, even if minute. In a different study using a forced reaction parameter of 8 and a computing time of $3 \times 10^{6} \mathrm{~s}, 94$ isotopes were included for this ${ }^{56} \mathrm{Fe}$ problem, of which 30 had relative errors below 1\%.) While this measured does provide quantitative information about the performance of the modeling scheme, its functional relationship to the number of simulated histories is unclear making it difficult to form a FOM that meets the other criteria: independent of number of histories and a one-to-one functional relationship with computational cost. This measure could be retained for comparing the efficiency of two schemes, but does not provide all the necessary information expected of a figure of merit. 


\subsubsection{Detection Limit $\left(1 / D_{k} T\right)$}

Often, an isotope with a relatively small inventory may have a disproportionate importance to the analysis in question due to some property of the isotope (e.g. its contribution to an engineering response such as the overall activity, the radiotoxicity or the waste disposal rating). The ability of a simulation to detect such a small existence of a particular isotope should also be taken into consideration when creating a FOM for an inventory problem. Moreover, since the specific isotope in question might not be known, it is important to use metric that provides information about the overall precision of the simulation. The detection limit can serve that purpose.

\section{Detection Limit in Monte Carlo Isotopic Inventory}

A $k$-percent detection limit $\left(D_{k}\right)$ is defined as the lowest relative production (yield) of all isotopes with relative error less than $k$ percent. Based on the definitions of the relative error and yield given by equations (12) and (13), the $k$-percent detection limit for an analog problem is therefore

$$
D_{k}=\frac{100^{2}}{N \cdot k^{2}}
$$

For a given a value of $\mathrm{k}$ the detection limit for analog Monte Carlo simulations is inversely proportional to $N$. However, this continuous $1 / N$ behavior implicitly assumes that the isotopic yields are continuous, that is, an uncountably infinite number of isotopes exist. This is certainly not the case for the Monte Carlo inventory method where the isotopic yields are distributed discretely.

For any results from a Monte Carlo isotopic inventory simulation, there can be only a finite number of isotopic products. The results will form a set of yields, $\left\{Y_{j}\right\}$, which can be sorted in descending order. At any point, the detection limit, $D_{k}$, will 
be defined by one of those isotopes, $i$, that has the smallest yield of all the isotopes with relative error less than $k$ percent. The isotope, $i+1$, has a smaller yield and a relative error larger than $k$ percent. As $N$ is increased, the relative error of both isotopes $i$ and $i+1$ will decrease $\left(R^{2} \propto 1 / N\right)$, but the detection limit will remain unchanged until the relative error of isotope $i+1$ drops below $k$ percent, at which point $D_{k}$ will drop suddenly to the value $Y_{i+1}$. The overall shape of the detection limit in a discrete problem should therefore be a series of steps where the leading edge follows the $1 / N(\propto 1 / T)$ dependence.

\section{Analyzing the detection limit in a real problem}

Figure 20 shows the $1 \%$ detection limits for an analog case and three different forced reaction parameters for the test problem. As expected, the detection limits for the analog case exhibits a $1 / T$ dependence. In addition to the step behavior caused by the discrete nature of the isotopic yields, the detection limits for the non-analog cases are all lower than that of the analog case and appear to depend similarly on $1 / T$. Power law curves were fit to the leading edge of each of these results to examine the relationship with $T$. The parameters of each fit are shown in the figure, where the multiplicative constant represents the relative improvement of the variance reduction method and the exponents are reasonably close to -1 . Once again, the discrete nature of the detection limit combined with the discrete time sampling of this test problem can contribute to an apparent departure from the $1 / T$ behavior.

The detection limit, $D_{k}$, can be used to evaluate the overall performance of the modeling scheme since a lower value $D_{k}$ indicates a greater precision in the answer. In Figure 20, orders of magnitude improvement are seen in the precision of the problem by invoking forced reaction variance reduction. Because of the $1 / T$ dependence of 


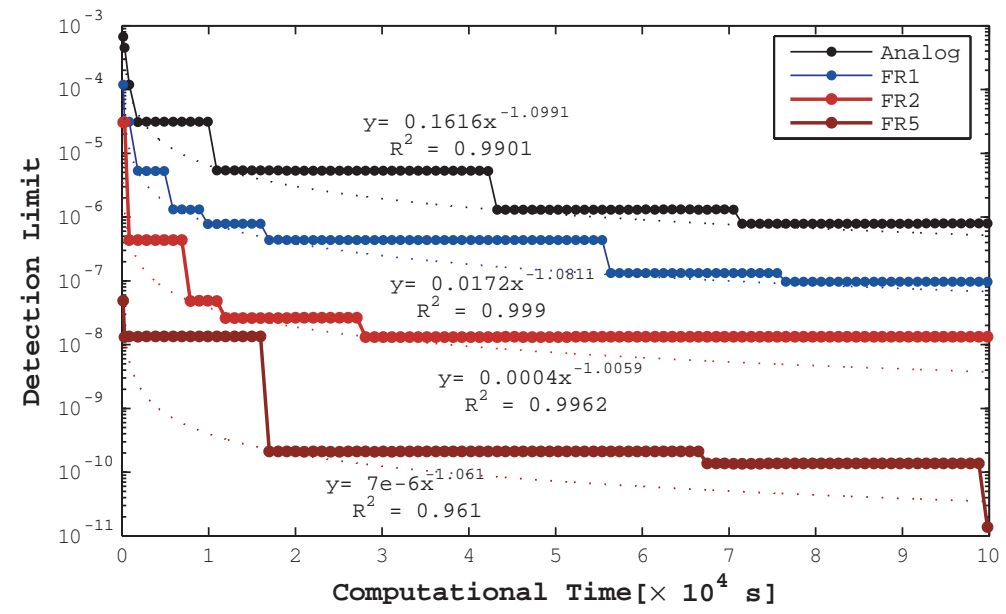

Figure 20: Detection limit as a function of computing time for an analog problem and three different forced reaction (FR) parameters. For each case, the points at the leading edge have been used to generate a power law fit whose parameters are also shown.

the detection limit, the most appropriate choice for a FOM is

$$
F O M=\frac{1}{D_{k} T}
$$

This FOM for the various cases of the test problem are shown in Figure 21. As expected, the overall shape of detection-limit-based FOM still exhibits the step behavior from $1 / D_{k}$. However, the $1 / T$ factor helps to lessen a strong step-function behavior and causes the FOM to oscillate about a constant value.

\subsubsection{Error Corrected Detection Limit $\left(1 / D_{k} R^{2} T\right)$}

The fluctuations in the detection limit based FOM are due to the $1 / T$ factor while the detection limit, $D_{k}$, is constant. Similarly, the relative error, $R_{i}$, of the isotope, $i$, that is defining the detection limit is declining continuously with time while that the detection limit is constant at that isotope's yield, $Y_{i}$. It should therefore be possible to mitigate the oscillatory behavior of the detection-limit-based FOM by using the 


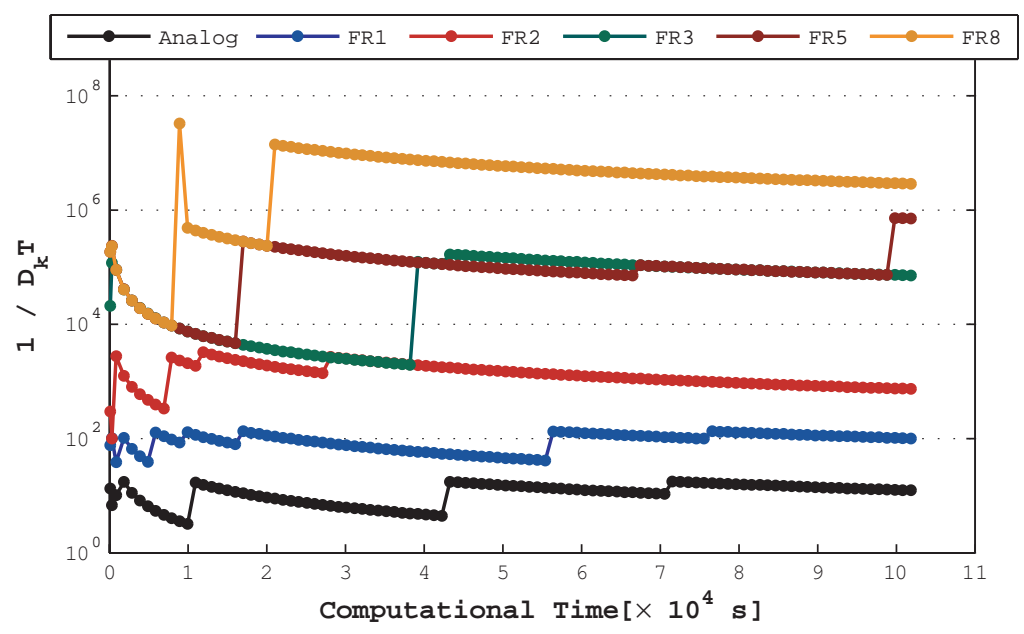

Figure 21: The detection limit based figure of merit is shown for the analog case and 5 different forced reaction (FR) variance reduction parameters.

relative error as a correction. In particular, it can be shown that a figure of merit, $1 / D_{k} R^{2} T$, is constant for the analog case. In regions where $D_{k}$ is constant, this FOM is constant since $R^{2}$ behaves as $1 / T$. Consider how $D_{k}$ and $R^{2}$ vary across a jump in the detection limit:

$$
\begin{array}{r}
\frac{D_{k}^{-}}{D_{k}^{+}} \equiv \frac{Y_{i}}{Y_{i+1}}=\frac{N_{i+1}}{N_{i}}=\frac{T_{i+1}}{T_{i}} \\
\frac{R_{-}^{2}}{R_{+}^{2}} \equiv \frac{R_{i}^{2}}{k^{2}}=k^{2} \frac{T_{i}}{T_{i+1}} \frac{1}{k^{2}}=\frac{T_{i}}{T_{i+1}} \\
\therefore D_{k}^{-} R_{-}^{2}=D_{k}^{+} R_{+}^{2}
\end{array}
$$

where $D_{k}^{-}$and $D_{k}^{+}$are the detection limit on the left and right of the jump, respectively, and $R_{-}^{2}$ and $R_{+}^{2}$ are the squares of the relative errors on the left and right of the jump, respectively. Thus, for analog problems this FOM is constant across a jump in the detection limit as well as during the periods where the detection limit does not change. 


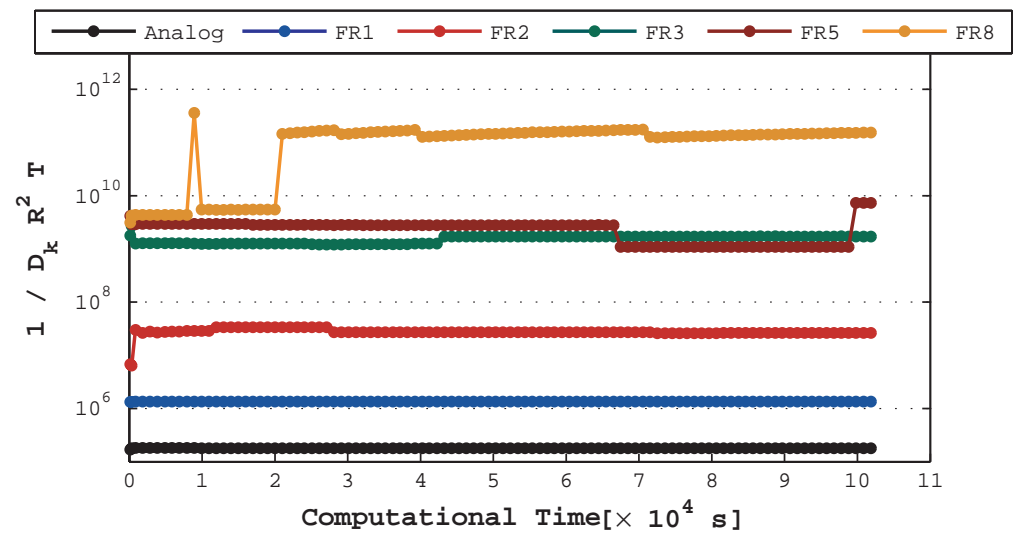

Figure 22: Figure of merit using error corrected detection limit provides a measure of the overall efficiency of the problem with a nearly constant value for a given modeling scheme.

Figure 22 shows results for an FOM with this formulation. The results agree with the analytic calculation that FOM is constant for analog case and nearly constant for the variance reduction cases. Even though, this FOM is likely to experience some fluctuations due to the discrete behavior of the detection limit, it can be used in a problem without a target isotope to compare the overall efficiency of different schemes. Most importantly, it is effective at providing an estimate of the necessary computing time for a given modeling technique to achieve a certain overall precision, as indicated by the detection limit.

\subsection{Summary}

A number of statistical measures can be employed to assess the performance of different Monte Carlo modeling schemes for a given isotopic inventory problem. From these, two valuable figures of merit have been derived that meet the criteria described above: a figure or merit should provide quantitative information about statistical performance, independent of the number of simulated histories, providing guidance on the relationship between computational cost and performance. The FOM based on 
the relative error of a single tally result $\left(1 / R^{2} T\right)$ is recommended for the problem with a known target isotope. In these situations, the modeling scheme can be optimized for that single result and the necessary computing time to achieve a target precision can be calculated. However, since many problems do not have readily identified target isotopes, or may have multiple important isotopes, a FOM based on the detection limit, corrected by the relative error, $\left(1 / D_{k} R^{2} T\right)$ is useful for assessing the overall efficiency of the problem. With this FOM, the modeling scheme can be optimized against the detection limit and then the necessary computing time to achieve a target detection limit can be calculated.

\section{$5 \quad$ Efficiency Assessment}

For each isotope in an analog Monte Carlo problem, the square of relative error is inversely proportional to a number of simulated particles and computing time. Hence, for every order of magnitude improvement in precision, two orders of magnitude more atoms must be supplied. Parallel computing is an immediate solution to reducing wall time to achieve a certain level of precision. Alternatively, variance reduction techniques are introduced to lower statistical error of a solution for the same number of Monte Carlo particles. However, this statistical gain comes at the expense of higher computing time per atom history. Thus, applying variance reduction techniques does not always provide the most efficient modeling scheme unless the statistical error of the result is reduced at a fast enough rate compared to the increase in computing time.

Figure of merit(FOM) can be used to quantitatively evaluate the efficiency of different Monte Carlo modeling schemes for the same physical problem and to provide useful guidelines to select the most efficient scheme, which sometimes may be a simple 
analog model.

\subsection{Problem Definition}

Monte Carlo simulations were designed to study efficiency variations resulting from different sets of variance reduction parameters and the use of FOM to monitor the efficiency. Several isotopic products, following a 10-year steady-state irradiation of ${ }^{56} \mathrm{Fe}$ under a uniform neutron flux of $5 \times 10^{12} \mathrm{n} / \mathrm{cm}^{2} \mathrm{~s}$, were selected such that their analog statistical errors were in distinctive ranges. Each chosen isotope was assumed to be an isotope of interest in separate study cases. Each study case was composed of many short test problems with different sets of variance reduction parameters. FOMs from short test problems were used to determine a set of variance reduction parameters that yielded the most efficient scheme for producing that particular isotope. The variance reduction techniques were limited to only biased reaction branching and forced reaction. A special case of the former, the so-called uniform reaction branching, was also considered. The biased reaction branching technique was in effect when the isotope of interest appeared as a product and the reaction probability density was adjusted according to the biasing score at the time. Otherwise, the uniform reaction branching technique was implemented to ensure that each reaction branch was equally followed and thus increase the possibility of capturing the isotope when it occurred deeply in rare reaction pathways. The number of simulated particles in each short test problem was $10^{6}$. The FENDL-2/A activation library and FENDL-2/D decay library were used in all cases. 


\subsection{Simulation Results}

Four isotopes, ${ }^{51} \mathrm{Cr},{ }^{49} \mathrm{Ti},{ }^{53} \mathrm{Mn}$ and ${ }^{60} \mathrm{Ni}$, with statistical errors of $100 \%, 37.80 \%$, $13.74 \%$ and $8.80 \%$, respectively, were chosen in the study from an analog problem with $10^{10}$ simulated particles. The reaction tree obtained from ALARA was used as a preliminary input for defining sets of variance reduction parameters to be tested. In addition, the testing sets were defined based on an assumption that the overall statistical quality of a particular isotope depends heavily on the qualities of the reaction branches that dominate the production of that isotope. Thus, improving statistical qualities of those branches was likely to subsequently better the statistical quality of the result.

The first study case was concerned with ${ }^{51} \mathrm{Cr}$ as the isotope of interest. ${ }^{51} \mathrm{Cr}$ was produced predominantly from the following reaction chain:

$$
{ }^{56} \mathrm{Fe} \rightarrow{ }^{53} \mathrm{Cr} \rightarrow{ }^{52} \mathrm{~V} \rightarrow{ }^{52} \mathrm{Cr} \rightarrow{ }^{51} \mathrm{Cr}
$$

The observed occurrence in the reaction tree of ${ }^{51} \mathrm{Cr}$ suggested a value of four for a forced parameter. Combinations of initial guessed values of forced parameter, 3, 4 and 5, and biasing scores of 5, 10 and 100 were used to construct a test set for finding the best modeling scheme for ${ }^{51} \mathrm{Cr}$.

According to Table 6, the test case when forced parameter and biasing score are 4 and 10, respectively, produced the highest value of FOM. It implied that this setup is the most efficient scheme for detecting ${ }^{51} \mathrm{Cr}$. Based on a previous analog problem with $10^{10}$ Monte Carlo particles, ${ }^{51} \mathrm{Cr}$ was produced with a relative error of $100 \%$, and a computational time of 5.58 hours. With the optimal scheme, the relative error of ${ }^{51} \mathrm{Cr}$ was improved to $5.28 \%$ while the computing time was reduced to 60 seconds. 


\begin{tabular}{|c|c|c|c|c|c|}
\hline \hline Case & $\begin{array}{c}\text { Forced } \\
\text { Parameter }\end{array}$ & $\begin{array}{c}\text { Biasing } \\
\text { Score }\end{array}$ & $\begin{array}{c}\text { Relative Error } \\
(\mathbf{\%})\end{array}$ & $\begin{array}{c}\text { FOM } \\
1 / R^{2} T\end{array}$ & $\begin{array}{c}\text { FOM } \\
1 / D_{1} R^{2} T\end{array}$ \\
\hline 0 & analog & analog & 100.00 & $4.98 \times 10^{-5}$ & $4.98 \times 10^{5}$ \\
\hline 1 & 3 & 5 & 8.95 & 2.4026 & $1.21 \times 10^{10}$ \\
\hline 2 & 4 & 5 & 11.16 & 1.1158 & $1.21 \times 10^{10}$ \\
\hline 3 & 5 & 5 & 32.42 & 0.1252 & $1.17 \times 10^{10}$ \\
\hline 4 & 3 & 10 & 9.93 & 2.2060 & $1.50 \times 10^{10}$ \\
\hline $\mathbf{5}$ & $\mathbf{4}$ & $\mathbf{1 0}$ & $\mathbf{5 . 2 8}$ & $\mathbf{5 . 9 7 4 6}$ & $\mathbf{1 . 1 5 \times 1 0 ^ { \mathbf { 1 0 } }}$ \\
\hline 6 & 5 & 10 & 5.04 & 5.1763 & $1.18 \times 10^{10}$ \\
\hline 7 & 3 & 100 & 15.79 & 0.9112 & $1.51 \times 10^{10}$ \\
\hline 8 & 4 & 100 & 13.35 & 0.9355 & $1.63 \times 10^{10}$ \\
\hline 9 & 5 & 100 & 11.21 & 1.0478 & $1.12 \times 10^{10}$ \\
\hline \hline
\end{tabular}

Table 6: The values of figure of merit $\left(1 / R^{2} T\right)$ were calculated from nine test runs and the analog problem $\left(10^{10}\right.$ particles for case 0$)$ when ${ }^{51} \mathrm{Cr}$ was a target isotope. The values of one-percent error corrected detection limit $\left(1 / D_{1} R^{2} T\right)$ were included to compare the general efficiencies of all test cases. One million simulated particles were used for each test problem.

Based on Equation (28), the test case would need about 67 seconds of computing time to produce ${ }^{51} \mathrm{Cr}$ with a relative error less than $5 \%$. The relative error from the analog case was statistically unreliable and could not be used to predict the computing time necessary to achieve a certain precision. The deterministic result of $8.420 \times 10^{-11}$ was assumed to be a theoretically correct result and used with Equation (29) to estimate a required number of particles to obtain a result with relative error less than $5 \%$. As a result, the analog problem would need about $4.80 \times 10^{12}$ particles translating to about $9.64 \times 10^{6}$ seconds of computing time.

It is interesting to consider an overall efficiency of the problem, which was designed to improve one particular result. The values of one-percent error corrected detection limit $\left(1 / D_{1} R^{2} T\right)$ were used to assess an overall efficiency of each test case. Relative magnitudes of those values implied that each test case performed at the same level of efficiency. This interpretation agreed with the actual physical modeling schemes, which had slight variations of biasing scores for the last reaction in each test case. As 


\begin{tabular}{|c|l|}
\hline \hline Target Isotope & Dominant Reaction Chains \\
\hline${ }^{49} \mathrm{Ti}$ & ${ }^{56} \mathrm{Fe} \rightarrow{ }^{52} \mathrm{Cr} \rightarrow{ }^{49} \mathrm{Ti}$ \\
& ${ }^{56} \mathrm{Fe} \rightarrow{ }^{53} \mathrm{Cr} \rightarrow{ }^{52} \mathrm{Cr} \rightarrow{ }^{49} \mathrm{Ti}$ \\
& ${ }^{56} \mathrm{Fe} \rightarrow{ }^{53} \mathrm{Cr} \rightarrow{ }^{50} \mathrm{Ti} \rightarrow{ }^{49} \mathrm{Ti}$ \\
\hline${ }^{53} \mathrm{Mn}$ & ${ }^{56} \mathrm{Fe} \rightarrow{ }^{55} \mathrm{Fe} \rightarrow{ }^{55} \mathrm{Mn} \rightarrow{ }^{54} \mathrm{Mn} \rightarrow{ }^{53} \mathrm{Mn}$ \\
& ${ }^{56} \mathrm{Fe} \rightarrow{ }^{55} \mathrm{Fe} \rightarrow{ }^{53} \mathrm{Mn}$ \\
& ${ }^{56} \mathrm{Fe} \rightarrow{ }^{55} \mathrm{Fe} \rightarrow{ }^{54} \mathrm{Fe} \rightarrow{ }^{53} \mathrm{Mn}$ \\
\hline${ }^{60} \mathrm{Ni}$ & $\rightarrow{ }^{57} \mathrm{Fe} \rightarrow{ }^{58} \mathrm{Fe} \rightarrow{ }^{59} \mathrm{Fe} \rightarrow{ }^{59} \mathrm{Ni}$ \\
& $\quad \rightarrow{ }^{50 m} \mathrm{Co} \rightarrow{ }^{60} \mathrm{Co} \rightarrow{ }^{60} \mathrm{Ni}$ \\
\hline
\end{tabular}

Table 7: Reaction channels leading to the most productions of ${ }^{49} \mathrm{Ti},{ }^{53} \mathrm{Mn}$ and ${ }^{60} \mathrm{Ni}$ were described and used for estimating the optimal values of variance reduction parameters.

expected, the most optimal scheme for calculating a result for one particular isotope was not the most efficient scheme when an overall efficiency of the problem was of interest.

As stated, biased reaction branching was not applied at a reaction where ${ }^{51} \mathrm{Cr}$ was not present as a product. This avoidance obviously introduced a degradation of performance in the model. Further increase in efficiency may be achieved with a more elaborately defined sets of variance reduction parameters for every reaction.

Table 7 shows the reaction pathways with the most productions of ${ }^{49} \mathrm{Ti},{ }^{53} \mathrm{Mn}$ and ${ }^{60} \mathrm{Ni}$. It is important to point out that uniform reaction branching was not used in the study case with ${ }^{60} \mathrm{Ni}$ because both of the product channels in the chains resulting in ${ }^{60} \mathrm{Ni}$ were extremely active and thus probable. The use of uniform reaction branching would, in fact, decrease frequency of ${ }^{60} \mathrm{Ni}$-dependent histories and consequently worsen the precision of ${ }^{60} \mathrm{Ni}$ 's detection.

The most efficient sets of forced parameter and biasing score for calculating the inventories of ${ }^{49} \mathrm{Ti},{ }^{53} \mathrm{Mn}$ and ${ }^{60} \mathrm{Ni}$ were summarized in Table 8 . With a simple application of variance reduction techniques, as large as four orders of magnitude 


\begin{tabular}{|c|c|c|}
\hline \hline Product & Force Parameter & Biasing Score \\
\hline${ }^{49} \mathrm{Ti}$ & 3 & 300 \\
\hline${ }^{53} \mathrm{Mn}$ & 3 & 100 \\
\hline${ }^{60} \mathrm{Ni}$ & 7 & 5 \\
\hline \hline
\end{tabular}

Table 8: The most efficient configurations of variance reduction parameters for detecting three isotopes in the study cases are shown.

improvement in efficiency was obtained in the study case of ${ }^{49} \mathrm{Ti}$. According to Table 9 , the results of ${ }^{49} \mathrm{Ti}$ and ${ }^{60} \mathrm{Ni}$ showed significant improvements in both statistical errors and computing time, with the statistical errors errors under two percent and computing time of 54 and 136 seconds, respectively. Although the statistical errors of ${ }^{53} \mathrm{Mn}$ was only improved slightly, the computing time for this studied isotope was greatly reduced from 5.58 hours to 54 seconds. The simulation results confirmed the initial guess that the optimal values of forced parameter were in the proximity of the position of the target isotope in the dominant reaction channels. For example, for ${ }^{49} \mathrm{Ti}$, the target isotope appeared 3 th and 4 th within the reaction channels, and the optimal forced parameter for ${ }^{49} \mathrm{Ti}$ is 3 . On the other hand, the most efficient choices of biasing score were determined on a trial-and-error basis. It was also worth noting that the effect of biased reaction branching tended to be insignificant in a case when a target isotope was the most active product. ${ }^{60} \mathrm{Ni}$ was produced predominantly from a $\beta^{-}$decay of ${ }^{60} \mathrm{Co}$. This reaction channel was far more active than others of ${ }^{60} \mathrm{Co}$. A high value of biasing score for this reaction channel would decrease the importance of each history due to weight adjustment and thus would not provide an efficient model. As a result, the optimal value of biasing score for ${ }^{60} \mathrm{Ni}$ turned out to be as low as 5 . 


\begin{tabular}{|c|c|c|c|c|c|c|}
\hline \hline \multirow{2}{*}{ Product } & \multicolumn{3}{|c|}{ Analog Problem } & \multicolumn{3}{c|}{ Study Case } \\
\cline { 2 - 7 } & $\begin{array}{c}\text { Relative } \\
\text { Error }\end{array}$ & $\begin{array}{c}\text { Comp. } \\
\text { Time (s) }\end{array}$ & F.O.M & $\begin{array}{c}\text { Relative } \\
\text { Error }\end{array}$ & $\begin{array}{c}\text { Comp. } \\
\text { Time (s) }\end{array}$ & F.O.M \\
\hline${ }^{51} \mathrm{Cr}$ & $100 \%$ & 20088 & 0.00005 & $5.28 \%$ & 60 & 5.97 \\
\hline${ }^{49} \mathrm{Ti}$ & $30.15 \%$ & 20088 & 0.00055 & $1.87 \%$ & 54 & 52.76 \\
\hline${ }^{53} \mathrm{Mn}$ & $13.13 \%$ & 20088 & 0.00290 & $11.08 \%$ & 54 & 1.51 \\
\hline${ }^{60} \mathrm{Ni}$ & $8.64 \%$ & 20088 & 0.00670 & $0.73 \%$ & 136 & 139.37 \\
\hline \hline
\end{tabular}

Table 9: Comparisons of relative error, computing time and FOM between an analog problem and study cases with optimal sets of variance reduction parameters are shown. Improvements in all areas from the analog problem are observed in all cases.

\subsection{Summary}

A reaction tree from a deterministic calculation was an important input for defining an initial set of variance reduction parameters to optimize the efficiency of production toward one particular isotope. It was shown that an optimal value of forced parameter depended on the position of that isotope in the reaction chain with the most relative production. Another factor that influenced the efficiency was biasing scores, which were more difficult to predict. The magnitude of biasing score of each reaction branch was likely to depend on its relative contribution toward that isotope's result. Improving the efficiency by all means would likely require a priori knowledge of a reaction tree, which sometimes can be computationally expensive.

\section{Summary}

This effort to develop Monte Carlo methods for isotopic inventory analysis was largely successful. While allowing for the exact modeling of complex flowing systems of irradiated material, there is a computational performance issue as those systems become more and more complex. The goals of the project were largely accomplished although sample calculations of real nuclear systems are still ongoing. Variance reduction tech- 
niques became a significant focus of the project in order to improve the overall modeling performance. Simple techniques to model chemical extraction processes were developed without the need for specialized chemical reaction models. A software tool is being made available for continued investigation of complex irradiation histories and benchmarking of deterministic approximations to those histories. 


\section{Publications}

- P. Phruksarojanakun, P.P.H. Wilson, "Monte Carlo Methods for Modeling Isotopic Inventories," Transactions of the American Nuclear Society, Vol 89, p 496, ANS, New Orleans, LA, USA (2003)

- P. Phruksarojanakun, P.P.H. Wilson, "Forced Reaction Variance Reduction for Monte Carlo Isotopic Inventory Methods," Transactions of the American Nuclear Society, Vol 90, ANS, Pittsburgh, PA, USA (2004)

- P. Phruksarojanakun, P. P. H. Wilson, "Figures of Merit in Monte Carlo Isotopic Inventory Methods," Proc. of Monte Carlo 2005, Chattanooga, TN (April 17$21,2005)$

- P. Phruksarojanakun, P. P. H. Wilson, "Biased Reaction Branching Variance Reduction for Monte Carlo Isotopic Inventory Methods," Transactions of the American Nuclear Society, Vol 93, Washington, DC, (2005)

- P. Phruksarojanakun, P. P. H. Wilson, "Efficiency of Variance Reduction Schemes for Monte Carlo Isotopic Inventory Analysis," Transactions of the American Nuclear Society, Vol 93, Washington, DC, (2005)

- P. P. H. Wilson and P. Phruksarojanakun, "Analog Monte Carlo Methods for Simulating Isotopic Inventories in Complex Systems", accepted for publication in Nuclear Science and Engineering, 2005

- P. Phruksarojanakun and P. P. H. Wilson, "Non-analog Implementation of Monte Carlo Isotopic Inventory Analysis," reviewed for publication in Nuclear Science and Engineering, 2006 


\section{References}

[1] P.P.H. Wilson. ALARA: Analytic and Laplacian Adaptive Radioactivity Analysis. PhD thesis, University of Wisconsin-Madison, 1999.

[2] R.F. Burstall. FISPIN - A Computer Code for Nuclide Inventory Calculations, October 1979. ND-R-328(R).

[3] R.A. Forrest and J-Ch. Sublet. FISPACT3 - Users Manual, April 1993. AEA/FUS 227.

[4] J. Jung. RACC: Theory and Use of the Radioactivity code RACC. Argonne National Laboratory, May 1979. ANL/FPP/TM-122.

[5] M.J. Bell. ORIGEN - The ORNL Isotope Generation and Depletion Code. Oak Ridge National Laboratory, May 1973. ORNL-4628.

[6] T.Y. Sung and W.F. Vogelsang. DKR: A Radioactivity Calculation Code for Fusion Reactors. University of Wisconsin - Fusion Technology Institute, September 1976. UWFDM-170.

[7] et al. T.R. England. CINDER-7: An Interim Report for Users. Los Alamos National Laboratory, April 1975.

[8] H. Bateman. Proc. cambridge phil. soc., 1910.

[9] J.F.E. Briesmeister. MCNP - A General Monte Carlo N-Particle Transport Code, Version 4C. Los Alamos National Laboratory, 2000.

[10] Iván Lux and László Koblinger. Monte Carlo Particle Transport Methods: Neutron and Photon Calculations. CRC Press. 
[11] M.H. Kalos and P.A. Whitlock. Monte Carlo Methods. J.Wiley and Sons, 1986.

[12] J.Spanier and E.M. Gelbard. Monte Carlo Principles and Neutron Transport Problems. Addison-Wesley Pub. Co., 1969.

[13] J.M. Hammersley and D.C. Handscomb. Monte Carlo Methods. Fletcher \& Son Ltd, 1964.

[14] P.W. Glynn and W.Whitt. The asymptotic efficiency of simulation estimators. Operations Research, 40(3), May-June 1992.

[15] Milad Fatenejad. Development and use of tools for modularization of activation programs. Undergraduate thesis, University of Wisconsin-Madison, 2005.

[16] The National Center for Supercomputing Applications. HDF5 User's Guide. University of Illinois, Mar 2005. 


\section{APPENDIX}

\section{A MCise Input File}

Extensible Markup Language (XML) has been implemented as a sole input file format [15] for MCise. It provides the users with the input format that is standardized and easy to use. For a given MCise calculation, an XML file is used as an input file to store the data the code needs to run. The input file is then parsed by any XML parsing libraries and validated with an XML schema file specifically written for MCise. The input file is composed of a series of elements. Each element can contain other elements and attributes that completely describe its characteristics. The definition of the element must be enclosed by a starting tag and an ending tag whereas the values of associated attribute are included inside the starting tag.

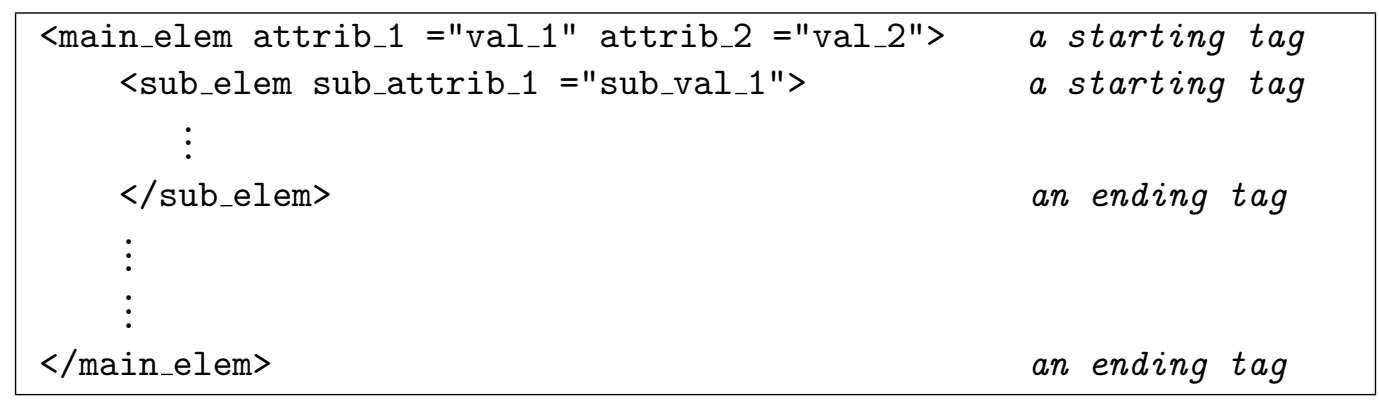

In general, there are 16 different types of main elements. Many elements can occur more than once, if necessary. However, all of the elements must appear in the order according to their appearances in the following sections. Comments are permitted and must be included between tags, " $<$ ! - -" and "-->".

\section{A.1 MCise}

MCise is the first element in the input file and also referred to as the root element. All subsequent main elements must be enclosed inside the root element. 


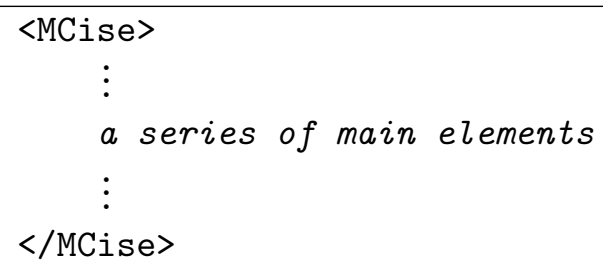

\section{A.2 NPS}

The NPS element specifies a number of Monte Carlo particles in the simulation. Its only attribute member is num.

$$
\text { <NPS num="10000" /> }
$$

\section{A.3 TotalTime}

This element define the total simulation time of each Monte Carlo history in seconds. It has only one attribute, time.

$$
<\text { TotalTime time="3600" / > }
$$

\section{A.4 TransLib and DecayLib}

The TransLib and DecayLib elements are used to define the locations of nuclear data library. The first one deals with the transmutation library while the second one involves with the library path to the decay library. Both paths are defined with respect to the current execution directory.

<TransLib path="/usr/local/data/fendlg-2.0_175" />

<DecayLib path="/usr/local/data/fendld-2.0" />

\section{A.5 WeightWindow}

Characteristic weight values of a weight window can be defined by the WeightWindow element. It has the following attributes. 
- lower defines a lower bound of the weight window.

- upper defines an upper bound of the weight window.

- target defines a weight of the particle that survives a Russian-roulette decision.

$$
\text { <WeightWindow lower="1e-10" upper="1.0" target="1e-5" /> }
$$

\section{A.6 FluxDef}

This element defines a set of neutron flux spectrums. Since different control volumes can have different flux spectrums, this element may occur as many times as necessary. It has one attribute, name that is a symbolic name, used to refer to this flux definition when a control volume is being created. In addition, it contains as many sub elements, Point, as necessary to represent the intensities of all energy groups. The order of the Point elements corresponds the energy bins, ranging from the first bin to the last bin. Each sub element point has two attributes which are value and repeat. The value attribute represents the intensity in $\frac{n}{b \cdot s}$. The repeat attribute is optional and represents a number of adjecent energy bins having the specified intensity. If it is not included, it will equivalent to assigning a value of "1" to repeat.

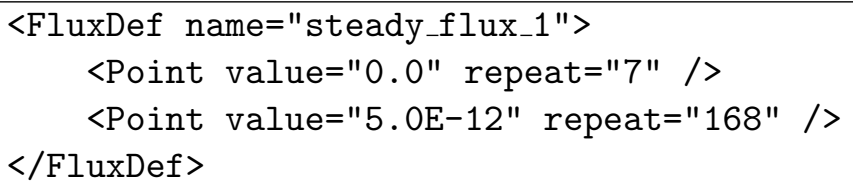

\section{A.7 Tally Elements}

Five different tally elements are designed to ensure that all of the types of tally and the natures of tally bin are captured. The tally elements, when created, are indepentdent of one another and may occur as many times as necessary to define all of the tallies 
used in the simulation. Each tally element has an attribute, name, to represent its symbolic name.

\section{A.7.1 ACT}

This element defines an atom current tally with one time plane in seconds specified by an attribute, time.

$$
<\text { ACT name="ac_1" time="3600" /> }
$$

\section{A.7.2 ACTEqualBin}

This element defines an atom current tally on a time range specified by attributes, low_bound and high_bound. An attribute, num_bins, is used to specify a number of time planes in the range. The time planes are equally separated from one another.

<ACTEqualBin name="ac_eq_1" num_bins="10" low_bound="0" high_bound="3600"/>

\section{A.7.3 APTEqualBin}

This element defines an atom population tally on a time range specified by attributes, low_bound and high_bound. The time range is equally divided into a certain number of bins defined by an attribute, num_bins.

<APTEqualBin name="ap_eq_1" num_bins="10" low_bound="0" high_bound="3600"/>

\section{A.7.4 ACTNonUniform}

This element defines an atom current tally with unequally separated time planes. Attributes, low_bound and high_bound, specify the range of the tally. Additional 
TimeInfo elements are used to define the relative time widths between each time plane.

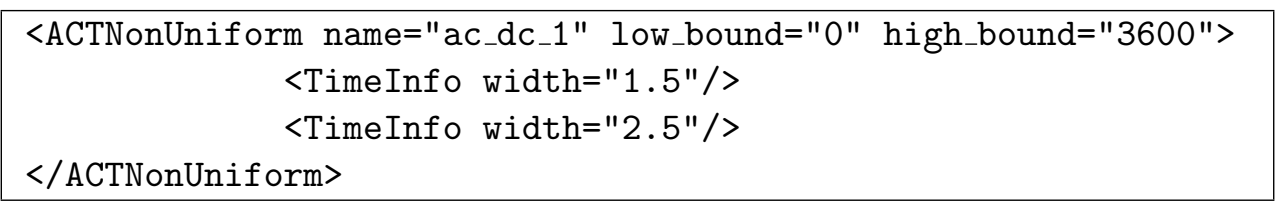

\section{A.7.5 APTNonUniform}

This element defines an atom population tally with non-uniform time bins. Attributes, low_bound and high_bound, specify the range of the tally. Additional TimeInfo elements are used to define the relative widths of the time bins.

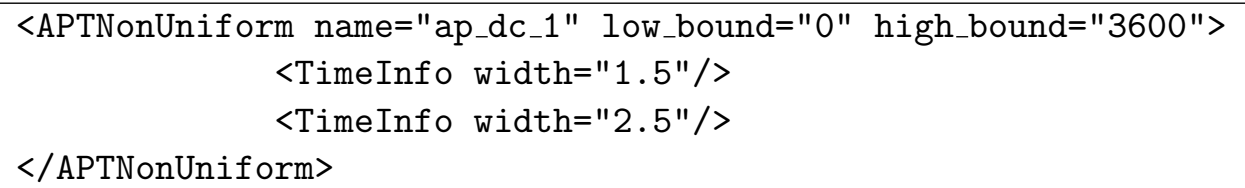

\section{A.8 ControlVolume}

The ControlVolume element consists of two attributes and four elements. An attribute, name, represents a symbolic name of the control volume and res_time defines the duration of the control volume in seconds. Both attributes are required for a complete definition of the control volume whereas some elements are not. The descriptions of the element members are the followings.

- Flux indicates a set of neutron flux to be used in the control volume. It contains only one attribute, name, which is a symbolic name declared by a FluxDef element. This element is required.

- Tally indicates a tally to be placed in the control volume. It contains only one attribute, name, which is a symbolic name declared by one of the tally 
elements. This element may occur as many times as a number of tallies in the control volume.

- $V R$ lists a variety of variance reduction techniques to be applied in the control volume. It does not have any attribute but consists of three optional sub elements.

- force indicates the use of a forced reaction technique. It has only one attribute, value, which represents a force parameter.

- Split contains one attribute, value, which is a boolean flag that determines whether or not a reaction path splitting technique is used in the control volume. The value accepts either "true" or "false".

- Biased enables a biased reaction branching technique by defining a biasing score for a specified isotope. Two attributes, $k z a$ and bias, correspond to the isotopic identity and a biasing score, respectively. This element can occur as often as necessary to include all of the isotopes which are needed to be biased and the biasing scores would be interpreted as relative scores among all Biased elements.

- CVExit is used to describe the material flowing path of the control volume. It contains two attributes, name which is a symbolic name of the next control volume or sink and probability which refers to a corresponding probability that the material would flow to this control volume. This element may be omitted if the current control volume is terminal and may occur as many times as necessary to capture all possibles flow paths. The probabilities will be automatically renormalized. Note that a biased reaction branching will have no effect if a reaction path splitting is applied. 


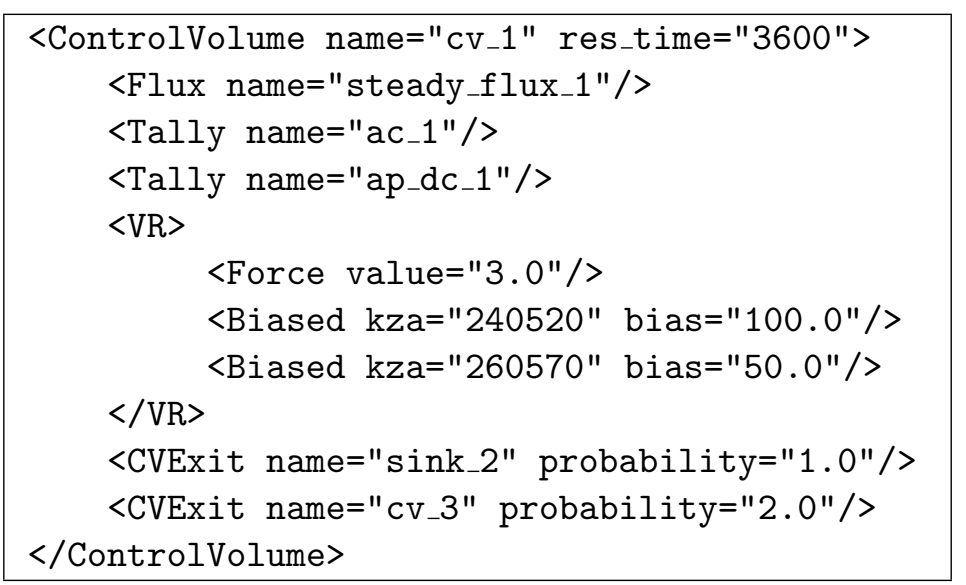

\section{A.9 Sink}

The Sink element contains two attributes, name and entrant_tally. The name attribute represents a symbolic name of this sink while the entrant_tally is a boolean flag that triggers the use of the atom entrant tally on the sink.

<Sink name="sink_1" entrant_tally="true"/>

\section{A.10 Mixture}

This element defines atomic compositions of a mixture. The Mixture element can occur more than once if there are multiple types of mixture in the simulation. It consists of one attribute, name, and one element, Atom. As usual, the name attribute represents a symbolic name of this mixture. The Atom element can occur as many times as necessary to complete a definition of this mixture. This element contains two attributes, $k z a$ and amount which represent the isotopic identity and its corresponding atomic percentage.

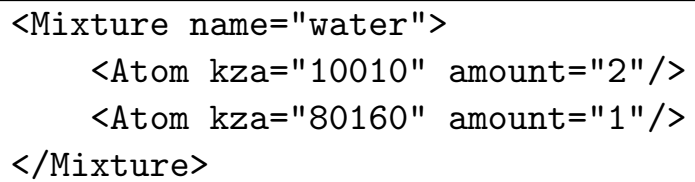




\section{A.11 Source}

This element is used to specify a source defintion. At least one of this element is required in the input file. It consists of the following required attributes.

- name represents a symbolic name of this source.

- mix indicates a mixture to be used as a source material. The mixture must be declared using the Mixture element.

- $c v$ accepts a symbolic name of the control volume to which this source is attached.

- time defines a simulation time in seconds when the source releases the material to the system.

- strength indicates a relative strength of this source. It is in effect when there is more than one source defined.

<Source name="sc_1" mix="water" cv="cv_1" time="0.0" strength="1.0"/>

\section{B MCise Output File}

Outputs from an MCise simulation are stored and organized in a file with a hierarchical data format known as HDF5 $5^{1}$. Generally, there are two primary objects, datasets and groups [16], in the HDF5 format. The first hierarchical level of the output file is consisted of one dataset, CPU time, and two groups of data, Reaction tree and Output tally. CPU time indicates the total computational time used in a particular run in seconds. Reaction tree contains a reaction tree information of the problem

\footnotetext{
${ }^{1}$ HDF5 is the latest development based on a HDF project which is developed and supported by the National Center for Supercomputing Applications at University of Illinois.
} 
whereas Output tally keeps output details of all tallies specified in the input file. Possible sub elements will also be described as their main elements are mentioned.

\section{B.1 Reaction tree}

For a given MCise simulation, only one reaction tree is generated. It contains the reaction pathway information of all isotopes in the simulation, regardless of when and where in the control volume they occur.

\section{B.1.1 Key}

A dataset Key indicates the isotopic identities of nodes in the reaction tree. Key is a column vector with indices and entries corresponding to node numbers and their isotopic identities, respectively. The initial material is always placed at the beginning of the reaction tree and designated with node number 0 . The isotopic identity of the initial material is also assigned to be 0 . Consequently, the Key vector always has 0 as its first entry.

\section{B.1.2 Parent}

A dataset Parent is a column vector containing the actual pathway information. Each index is regarded as one daughter node and its parent node is given by the entry associated with the index. The parent node of the initial material is defined to be 0 by default. As a result, the first entry of the Parent vector that reflects the parent node of the initial material is always 0 .

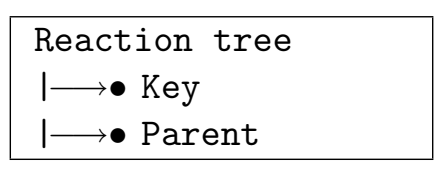




\section{B.2 Output tally}

A group Output tally does not contain any dataset; however, it has as many subgroups as a number of tallies declared in the input file.

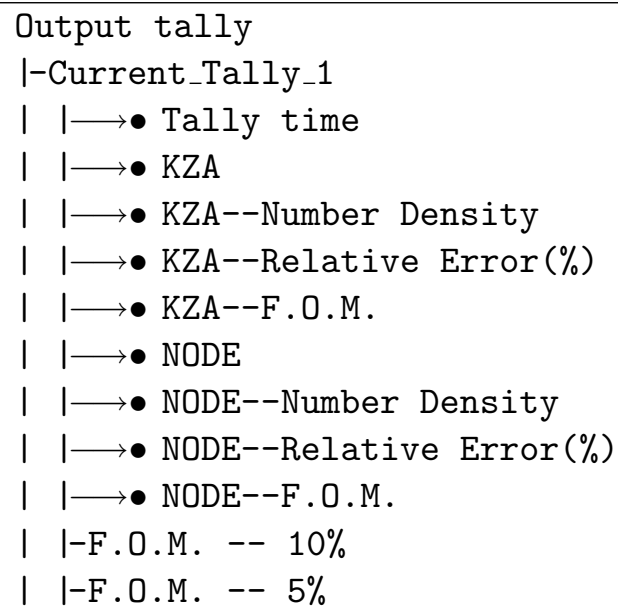

Each tally group contains nine datasets and two groups.

\section{B.2.1 Tally time}

A dataset Tally time is a column vector that contains the time information in seconds of a tally and is directly associated with a number of tally bins. AtomCurrentTally has the same number of tally bins as the size of the Tally time vector while AtomPopTally has one less tally bin than the size of the same vector.

\section{B.2.2 A KZA category}

Datasets with their name starting with KZA belong to this category. Their descriptions are described below.

\section{KZA}

A dataset KZA is a column vector that contains all of the isotopic identities whose existences are detected by a particular tally. The KZA vector is used as a reference 
column for other characteristics outputs that belong to a category KZA.

KZA--Number Density

A dataset KZA--Number Density is a matrix with numbers of columns and rows corresponding to the size of the KZA vector and a number of tally bins. Each entry in the matrix represents the production of a given isotope with respect to a unit atomic amount of the initial material.

KZA--Relative Error $(\%)$

A dataset KZA-Relative Error $(\%)$ is a matrix with the same dimensions as a dataset KZA--Number Density. It contains relative errors of the results.

KZA--F.O.M.

A dataset KZA--F.O.M. is a matrix containing figures of merit of every entry in the KZA vector for all tally bins. The dimensions of this matrix are the same as Number Density.

\section{B.2.3 A NODE category}

Instead of being sorted by KZA, the results are also organized according to node numbers from the Reaction tree. This set of output provides finer details of the inventory productions as it suggests relative contributions from each reaction point in the Reaction tree. The output format of this category is virtually the same as a previous one. 


\section{B.2.4 General F.O.M.}

The overall efficiency of the simulation suggested by a particular tally is given in terms of FOM based on the detection limit, corrected by the relative error $\left(1 / D_{k} R^{2} T\right)$. The default values for $k$ are 5 and 10 percent. For each default value, there are three datasets: KZA, Relative Error(\%) and F.0.M.. KZA specifies an isotope with the lowest relative production and Relative Error $(\%)$ less than $k$ percent. Finally, F.O.M. is the resulting figure of merit.

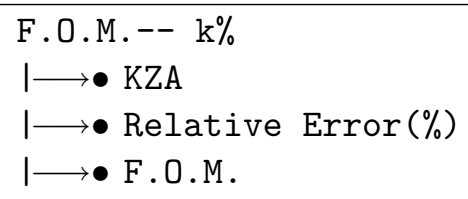

